\title{
L-DOPA sensitizes vasomotor tone by modulating the vascular alpha1-adrenergic receptor
}

Daiki Masukawa, ${ }^{1}$ Motokazu Koga, ${ }^{1}$ Anna Sezaki, ${ }^{1,2}$ Yuka Nakao, ${ }^{1}$ Yuji Kamikubo, ${ }^{3}$

Tatsuo Hashimoto, ${ }^{1,4}$ Yuki Okuyama-Oki, ${ }^{4}$ Aderemi Caleb Aladeokin, ${ }^{1}$ Fumio Nakamura, ${ }^{1}$ Utako Yokoyama, ${ }^{5}$ Hiromichi Wakui, ${ }^{4}$ Hiroshi Ichinose, ${ }^{2}$ Takashi Sakurai, ${ }^{3}$ Satoshi Umemura, ${ }^{4}$ Koichi Tamura, ${ }^{4}$ Yoshihiro Ishikawa, ${ }^{5}$ and Yoshio Goshima ${ }^{1}$

'Department of Molecular Pharmacology and Neurobiology, Yokohama City University Graduate School of Medicine, Yokohama, Japan. ${ }^{2}$ Graduate School of Bioscience and Biotechnology, Tokyo Institute of Technology, Yokohama, Japan. ${ }^{3}$ Department of Pharmacology, Juntendo University School of Medicine, Tokyo, Japan. ${ }^{4}$ Medical Science and Cardiorenal Medicine, and ${ }^{5}$ Cardiovascular Research Institute, Yokohama City University Graduate School of Medicine, Yokohama, Japan.

Blood pressure is regulated by extrinsic factors including noradrenaline, the sympathetic neurotransmitter that controls cardiovascular functions through adrenergic receptors. However, the fine-tuning system of noradrenaline signaling is relatively unknown. We here show that L-3,4dihydroxyphenylalanine (L-DOPA), a precursor of catecholamines, sensitizes the vascular adrenergic receptor alpha1 (ADRA1) through activation of L-DOPA receptor GPR143. In WT mice, intravenous infusion of the ADRA1 agonist phenylephrine induced a transient elevation of blood pressure. This response was attenuated in Gpr143 gene-deficient (Gpr143-/y) mice. Specific knockout of Gpr143 in vascular smooth muscle cells (VSMCs) also showed a similar phenotype, indicating that L-DOPA directly modulates ADRA1 signaling in the VSMCs. L-DOPA at nanomolar concentrations alone produced no effect on the VSMCs, but it enhanced phenylephrine-induced vasoconstriction and intracellular $\mathrm{Ca}^{2+}$ responses. Phenylephrine also augmented the phosphorylation of extracellular signal-regulated kinases in cultured VSMCs from WT but not Cpr143-/y mice. In WT mice, blood pressure increased during the transition from light-rest to dark-active phases. This elevation was not observed in Gpr143-/y mice. Taken together, our findings provide evidence for L-DOPA/GPR143 signaling that exerts precursor control of sympathetic neurotransmission through sensitizing

Conflict of interest: The authors have declared that no conflict of interest exists.

Submitted: October 13, 2016 Accepted: August 15, 2017 Published: September 21, 2017

Reference information: JCI Insight. 2017;2(18):e90903. https://doi.org/10.1172/jci. insight.90903. vascular ADRA1.

\section{Introduction}

L-3,4-dihydroxyphenyalanine (L-DOPA), a precursor of catecholamines, is considered an inert amino acid (1), and it ameliorates the symptoms of Parkinson's disease following its conversion to dopamine in the brain by aromatic L-amino acid decarboxylase (AADC). In contrast to this generally accepted idea, we propose a transmitter role for L-DOPA by itself. L-DOPA is released from the brain, sympathetic nerve terminals, or adrenal medulla in vivo in a $\mathrm{Ca}^{2+}$-dependent (2-4) and tetrodotoxin-sensitive (voltage-dependent sodium channel blocker) manner $(2,5)$. L-DOPA probably plays a neurotransmitter role in the primary baroreceptor afferents terminating in the nucleus tractus solitarii (NTS) $(5,6)$. When L-DOPA is microinjected into the NTS, it produces a depressor and bradycardic response that is not modified by AADC inhibition (7); however, the response is antagonized by L-DOPA cyclohexyl ester (L-DOPA CHE), an antagonist for L-DOPA $(5,6)$. In the depressor sites of the NTS, some neurons are tyrosine hydroxylase (TH) and L-DOPA positive but AADC and dopamine negative (6). Aortic nerve stimulation induces reflex bradycardia and a concomitant release of L-DOPA from the NTS (6).

We recently identified GPR143 as a receptor for L-DOPA that mediates the depressor response to L-DOPA in the NTS (8). GPR143 is expressed in the NTS, and RNAi knockdown of GPR143 suppresses the depressor response to L-DOPA microinjected into the NTS. In addition, specific, saturable, and L-DOPA CHE-displaceable $\left[{ }^{3} \mathrm{H}\right]$-DOPA binding sites were detected in GPR143-expressing CHO cells (8). GPR143 
was identified as the protein product of the oal gene, and mutation of the oal gene causes ocular albinism type 1 , characterized by severe reduction of visual acuity, optic misrouting, and hypopigmentation of the retinal pigment epithelium $(9,10)$. GPR143 possesses L-DOPA-binding activity and could function as an L-DOPA receptor in retinal pigment epithelium cells (11). Although the response to L-DOPA was characterized in in vitro reconstitution experiments, the mechanisms and functions of GPR143 in vivo still remain obscure (11).

GPR143 is also expressed in non-neuronal organs receiving sympathetic innervation, such as the heart and blood vessels (12), thereby suggesting its involvement in peripheral cardiovascular regulation. Therefore, to further determine whether GPR143 plays a physiological role, we examined peripheral vasomotor response to i.v. administration of phenylephrine, a nonselective adrenergic receptor, alpha1 (ADRA1) agonist, in GPR143 gene-deficient (Gpr143-/y) mice (12). We found that the pressor response to phenylephrine was markedly attenuated in $G$ pr $143^{-1 y}$ mice compared with $G p r 143^{+/ y}$ (WT) mice. The contractile response to phenylephrine in isolated blood vessels from $G$ pr $143^{-/ y}$ mice was attenuated compared with the response in WT mice. The rise in blood pressure response to nociceptive stimulation and dark-active phase, which

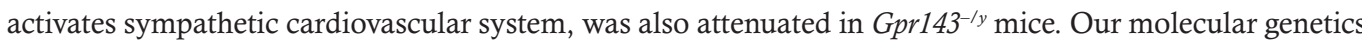
and phenotypic analyses reveal that L-DOPA/GPR143 signaling functionally couples with ADRA1 and regulates sympathetic neurotransmission in the cardiovascular system.

\section{Results}

GPR143 in vascular smooth muscle cells is involved in phenylephrine-induced blood pressure rise in mice. To determine the in vivo function of GPR143 in the peripheral cardiovascular system, we first examined the response to

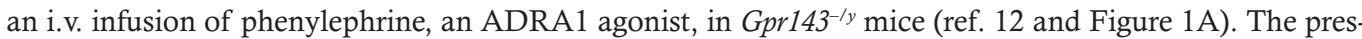
sor response to phenylephrine in itself was attenuated in $G p r 143^{-1 y}$ mice compared with WT mice (Figure 1, B and C). Pretreatment with L-DOPA CHE, a competitive antagonist for GPR143 (8), also attenuated the pressor response to phenylephrine (Figure 1D). We also compared the effects of vasopressin, which caus-

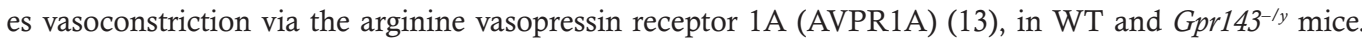

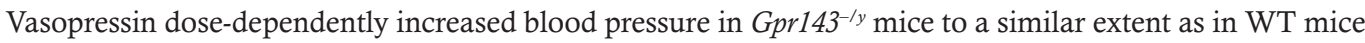
(Figure 1E), suggesting that GPR143 is selectively involved in the ADRA1-mediated response. Because vascular tone is regulated by ADRA1, we investigated whether GPR143 was expressed in vascular smooth muscle cells (VSMCs). GPR143 immunoreactivities showed a dot-like appearance in the cytoplasm of the mouse VSMCs. The dot-like appearance of GPR143-immunoreactive signals may reflect its localization in late endosome/lysosome, as previously described (14). GPR143 also localized in the plasma membrane of RPE and COS-7 cells $(11,15)$. Some signals were also detected in the plasma membrane. No immunoreactive signals were observed in Gpr $143^{-1 y}$ mice, confirming the expression of GPR143 in the VSMCs (Supplemental Figure 1; supplemental material available online with this article; https://doi.org/10.1172/ jci.insight.90903DS1). To determine whether attenuation of the pressor response to phenylephrine was due to the loss of VSMC GPR143, we generated conditional knockout mice deficient in GPR143 in the

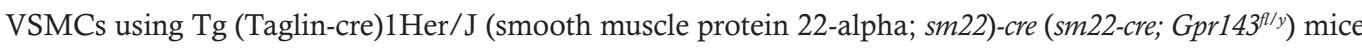
(16). Again, the pressor response to phenylephrine was attenuated in sm22-cre; Gpr $143^{f / y}$ mice compared with sm22-cre; Gpr143 ${ }^{W T}$ mice (Figure 1F), thereby indicating that the cardiovascular defect was caused by the GPR143 loss of function expressed in VSMCs. These phenotypic defects were unlikely to be due to possible alterations in the expression levels of receptors involved in blood pressure control. In fact, mRNA levels of Adra1 subtypes in descending aortas were not altered in $G p r 143^{-/ y}$ mice compared with WT mice. The fold changes in the gene expression levels in the Gpr143-/y mice relative to those in the WT mice were $1.03 \pm 0.128$ for Adra1a, $1.06 \pm 0.31$ for Adra1b, and $1.00 \pm 0.21$ for Adra1d $(n=6, P>0.05$, unpaired Student's $t$ test). It is also unlikely that the attenuated response to phenylephrine in $G p r 143^{-1 y}$ mice was due to decreased levels of endogenous L-DOPA, since no differences existed in the expression levels of TH in sympathetic neurons in the descending aortas and adrenal glands or in the plasma levels of L-DOPA in Gpr143- ${ }^{-1 y}$ and WT mice (Supplemental Figure 2). These findings suggest that GPR143 expressed in VSMCs plays a role in the regulation of vascular responsiveness to phenylephrine.

Sm22-cre is also functional in cardiomyocytes (17). To elucidate possible involvement of GPR143 expressed in cardiomyocytes, we also investigated chronotropic responses to phenylephrine and isoproterenol, an ADRB1 agonist in isolated atrial preparations. Both phenylephrine $(10 \mathrm{nM}-100 \mu \mathrm{M})$ and isoproterenol (10 pM-100 nM) concentration-dependently increased the heart rates. The increase in heart rate mediated by phenylephrine and isoproterenol was similar in WT and Gpr143-/y atria (Supplemental Figure 4). 
A

$\mathrm{a}_{1}$ AR agonist;

Phenylephrine (i.v.)
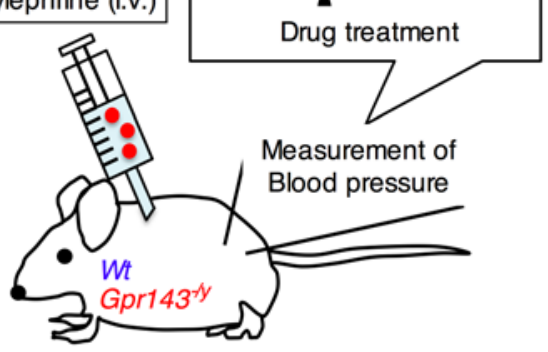

D

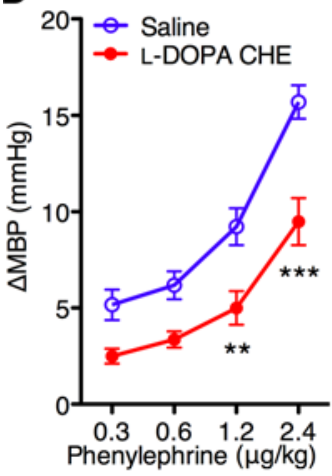

E

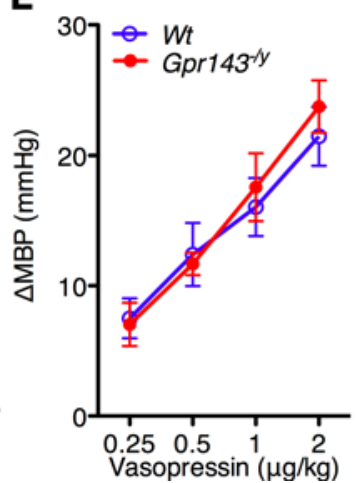

B
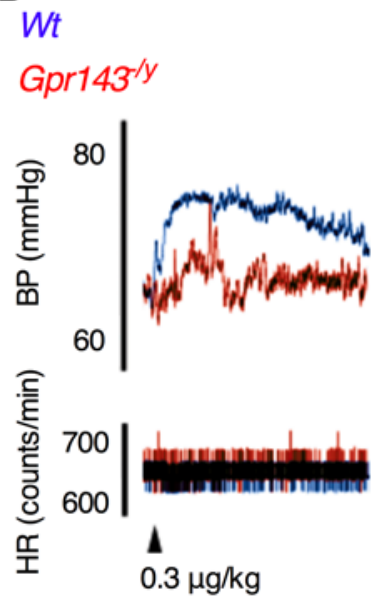

$\mathbf{F}$

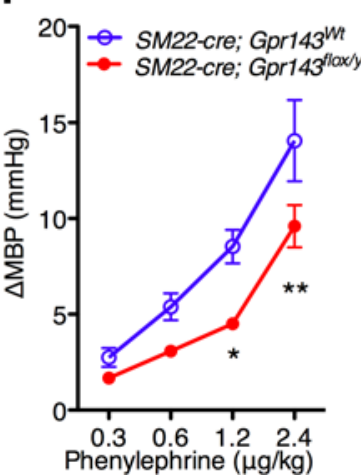

C
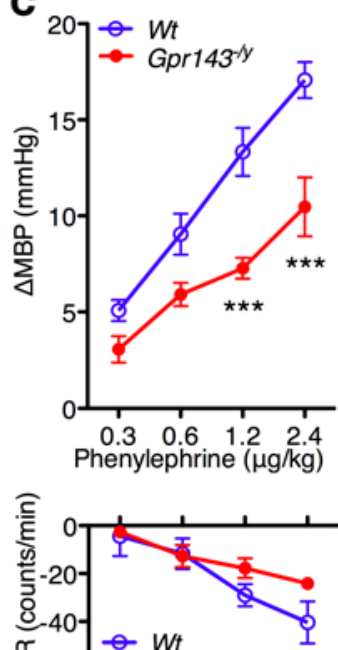

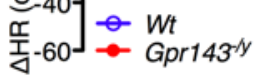

G

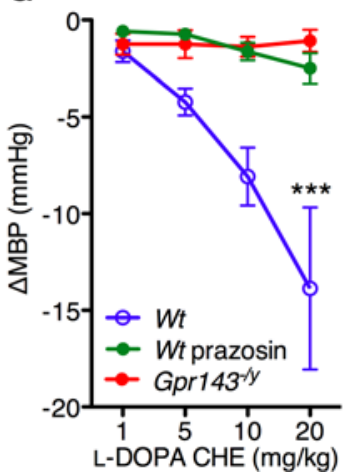

Figure 1. Impaired pressor response to phenylephrine in Gpr143-/y mice. (A) Schematic representation of the experimental design. Measurement of blood pressure (BP) and heart rate (HR) was performed on anesthetized mice, and drugs were administered i.v. (B) Typical traces of the effects of phenylephrine on $\mathrm{BP}$

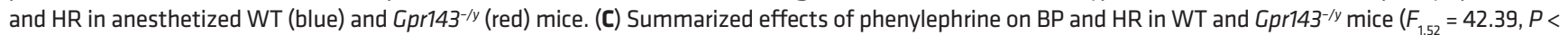
$0.001, n=7$ and 8). (D) Effects of phenylephrine on BP under pretreatment with saline or L-DOPA cyclohexyl ester (CHE) $(10 \mathrm{mg} / \mathrm{kg})\left(F_{1,40}=46.75, P<0.001, n=6\right)$. (E) Effects of vasopressin on BP in WT and Gpr143- ${ }^{-1 y}$ mice $\left(F_{132}=0.209, P>0.05, n=5\right)$. (F) Effects of phenylephrine on BP in SM22-cre; Gpr143 ${ }^{W T}$ and SM22-cre; Gpr143 ${ }^{f / y}$ mice $\left(F_{1,44}=20.99, P<0.005, n=6\right.$ and 7). (C) Effects of L-DOPA CHE alone on BP in WT mice with or without prazosin (1 mg $/ \mathrm{kg}$, i.p.) and in Cpr143 ${ }^{-1 / y}$ mice $\left(F_{1,28}=10.18, P<0.005, n=4-7\right)$. All values are mean \pm SEM. ${ }^{*} P<0.05,{ }^{* *} P<0.01,{ }^{* * *} P<0.001,2$-way ANOVA with Bonferroni's multiple comparisons test.

To elucidate a tonic function of GPR143, we investigated an acute effect of L-DOPA CHE on blood pressure in Gpr $143^{-1 y}$ and WT mice. i.v. infusion of L-DOPA CHE alone dose-dependently decreased the basal levels of mean blood pressure in WT mice. The action of L-DOPA CHE was suppressed by pretreatment with prazosin ( $1 \mathrm{mg} / \mathrm{kg}$, i.p.), an ADRA1 antagonist. Furthermore, the depressor action of L-DOPA CHE alone was lacking in Gpr143-1/ mice (Figure 1G). Furthermore, prazosin bound to ADRA1B, but not to GPR143 (Supplemental Figure 3). This finding indicates that GPR143 is tonically activated via ADRA1 in vivo and is involved in maintaining vasomotor tone.

L-DOPA augments the contractile and intracellular $\mathrm{Ca}^{2+}$ signal responses to phenylephrine through GPR143. We investigated phenylephrine-induced vasoconstriction in the descending aortas and mesenteric resistance

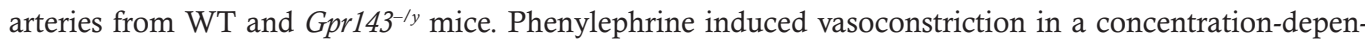
dent manner in WT aortas and mesenteric arteries. The effect of phenylephrine was markedly attenuated

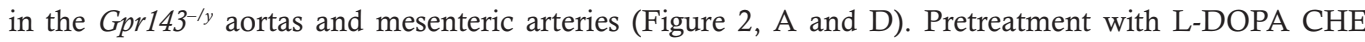
suppressed the effect of phenylephrine in WT aortas (Figure 2B). L-DOPA $(10 \mathrm{nM})$ alone had no effect on vasocontractility in WT aortas and mesenteric arteries. Non-effective L-DOPA $(0.1,1$, and $10 \mathrm{nM})$, when pretreated, augmented the vasoconstricting effect of phenylephrine (100 $\mathrm{nM}$ and $1 \mu \mathrm{M})$ in WT aortas and mesenteric arteries (Figure 2, C and E). Consistent with this finding, L-DOPA-induced augmentation was

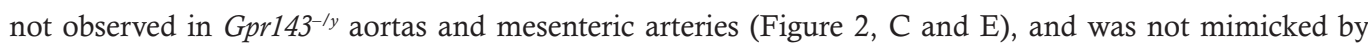
dopamine $(10 \mathrm{nM})$ in WT aortas (Figure 2C). Again, L-DOPA did not modify the contractile response 
A

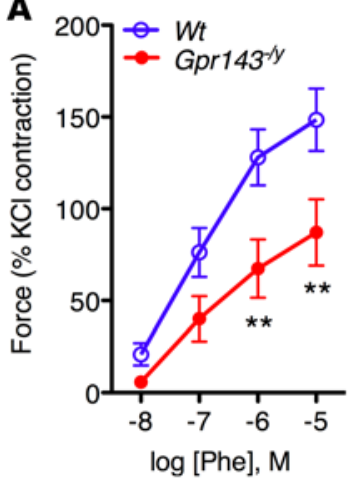

D

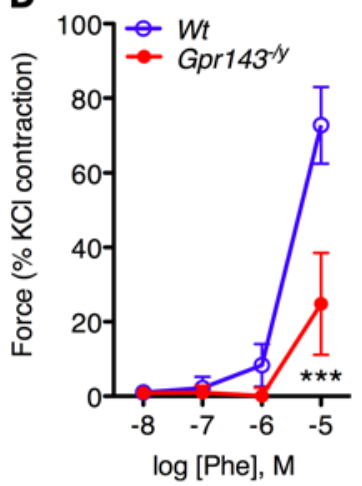

B
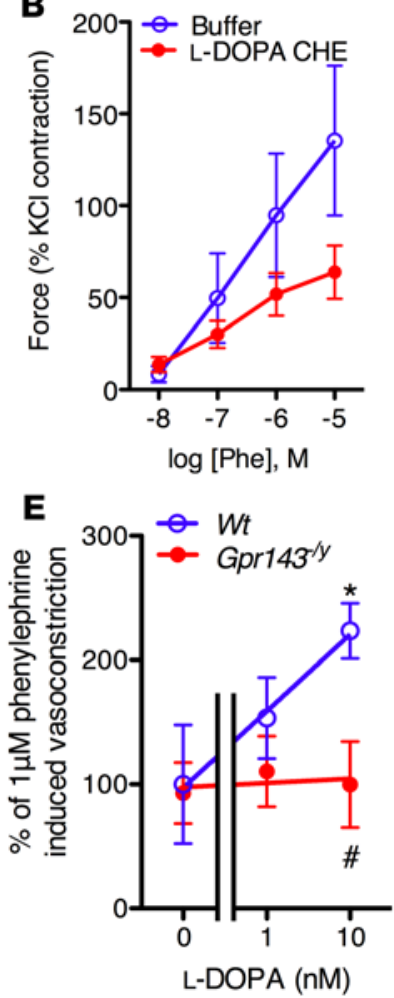
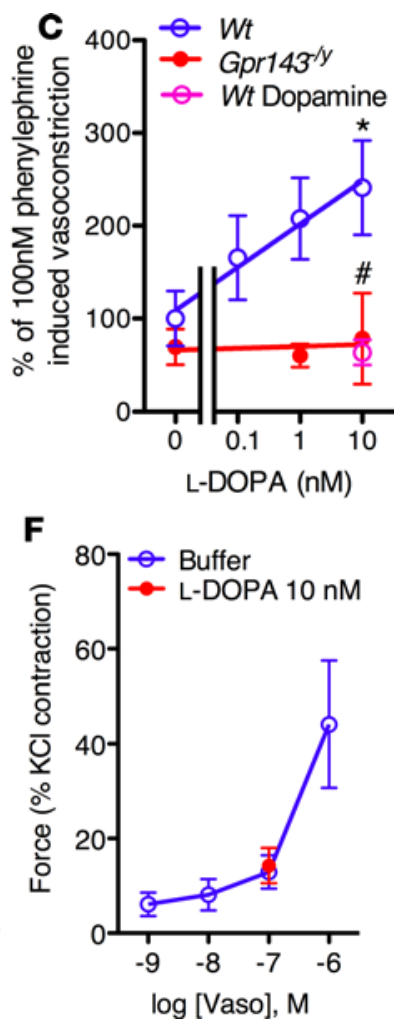

Figure 2. L-DOPA augments the vasocontractile response to phenylephrine through GPR143. Contractile response in dissected aorta. (A) Contractile responses to phenylephrine (Phe) in WT and Gpr143-/y aortas $\left(F_{1,68}=20.29, P<0.001, n=9\right.$ and 10). (B) Contractile response to phenylephrine, combined with control buffer or L-DOPA cyclohexyl ester (CHE) ( $1 \mathrm{mM})$ in WT aortas $\left(F_{1,56}=4.363\right.$, $P<0.01, n=8)$. (C) Contractile response to phenylephrine $(100 \mathrm{nM})$ in the presence of increasing concentrations of L-DOPA in WT and Gpr143-1/y aortas $\left(F_{1,45}=10.16, P<0.01, n=6-11\right)$. Contractile response in dissected mesenteric arteries. (D) Contractile response to phenylephrine in WT and Cpr143 $^{-1 y}$ arteries $\left(F_{1,44}=9.684, P<0.001, n=6\right)$. (E) Contractile response to phenylephrine $(1 \mu \mathrm{M})$ in the presence of increasing concentration of L-DOPA in WT and Gpr143 ${ }^{-/ y}$ arteries $\left(F_{1,38}=4.565\right.$, $P<0.05, n=6-11)$. (F) Contractile response to vasopressin (Vaso) in WT and Gpr143- ${ }^{-1 y}$. L-DOPA $(10 \mathrm{nM})$ did not augment the effect of vasopressin $(n=6)$. Contractility is expressed as the percentage of the contraction induced by $\mathrm{KCl}(10 \mathrm{mM})$. ${ }^{*} P<0.01$, ${ }^{* *} P<0.001$, compared with WT (A and D). ${ }^{*} P<0.05$, compared with $0 \mathrm{nM} \mathrm{L-DOPA}$ in WT (C and E), 1-way ANOVA with Dunnett's multiple comparisons test. ${ }^{*} P<0.05$, compared with $10 \mathrm{nM} \mathrm{L-DOPA}$ in WT (C and $\mathbf{E}$ ), 2-way ANOVA with Bonferroni's multiple comparisons test.

to vasopressin in WT mesenteric arteries (Figure $2 \mathrm{~F}$ ). The effective concentration range of L-DOPA $(\sim 10$ $\mathrm{nM}$ ) was comparable to the plasma concentration range observed in vivo. In WT mice, the plasma concentrations of L-DOPA in light and dark phases were $989.7 \pm 178.1 \mathrm{pg} / \mathrm{ml}(5.0 \pm 0.90 \mathrm{nM})$ and 2,180.0 \pm $529.8 \mathrm{pg} / \mathrm{ml}(11.1 \pm 2.7 \mathrm{nM})$, respectively $(n=9-13)$ (Supplemental Figure $2 \mathrm{C})$.

We also examined intracellular $\mathrm{Ca}^{2+}$ response in VSMCs. Phenylephrine $(1-100 \mu \mathrm{M})$ and vasopressin (1.5-150 nM) concentration-dependently increased the intracellular $\mathrm{Ca}^{2+}$ in WT and Gpr143-ly VSMCs (Figure 3, A and B). L-DOPA (1-10 nM) produced no effect on $\mathrm{Ca}^{2+}$ levels, but it enhanced the responses to phenylephrine, but not the responses to vasopressin (Figure 3, C-E). Again, L-DOPA failed to enhance

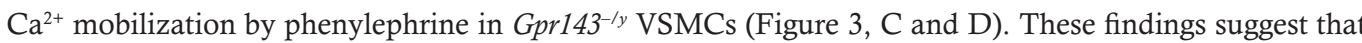
L-DOPA sensitizes the response of ADRA1s through GPR143.

Using a peptide derived from the porcine teschovirus sequence with high cleavage efficiency in two proteins (18), we reconstructed the polycistronic ADRA1B and GPR143 expression plasmid in ARPE19, a human retinal pigment epithelial cell line. In the ARPE19 cells expressing ADRA1B-Myc and GPR143-mCherry (Supplemental Figure 5, A and B), phenylephrine (30 pM to $10 \mathrm{nM}$ ) induced an increase in the intracellular $\mathrm{Ca}^{2+}$ response, which was antagonized by pretreatment with $1 \mu \mathrm{M}$ prazosin. The ineffective concentration of phenylephrine was estimated to be $30 \mathrm{pM}$ (Supplemental Figure 5, C and D). L-DOPA (1-30 $\mathrm{nM})$ augmented the effect of the $\mathrm{Ca}^{2+}$ increase induced by phenylephrine ( $\left.30 \mathrm{pM}\right)$, which was antagonized by L-DOPA CHE (100 nM to $10 \mu \mathrm{M}$ ) (Supplemental Figure 5, E and F). The $\mathrm{EC}_{50}$ for the augmentation by L-DOPA was estimated to be $5.7 \mathrm{nM}(n=3-12)$. The $\mathrm{EC}_{50}$ value for L-DOPA was again comparable to its plasma concentration range observed in vivo. We then examined whether phenylephrine augments the $\mathrm{Ca}^{2+}$ response to L-DOPA. As in the case with VSMCs (Figure 3), L-DOPA up to $10 \mu \mathrm{M}$ alone did not induce $\mathrm{Ca}^{2+}$ response. Unlike L-DOPA, pretreatment with an ineffective concentration of phenylephrine (30 pM) did not modify intracellular $\mathrm{Ca}^{2+}$ levels after the application of L-DOPA (Supplemental Figure 5, G-J). These findings suggest that L-DOPA activates GPR143, thereby modulating the functions of ADRA1B, while phenylephrine activates ADRA1B without modifying the function of GPR143.

Phenylephrine-induced myosin light chain 2 and ERK phosphorylations were attenuated in Gpr143-ly blood vessels and VSMCs. To further investigate the mechanisms underlying inhibition of phenylephrine-induced vascular contraction by deletion of GPR143, we also examined phosphorylation levels of myosin light chain

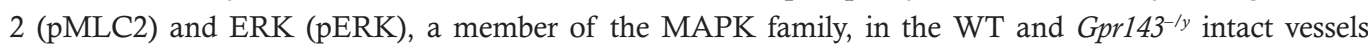



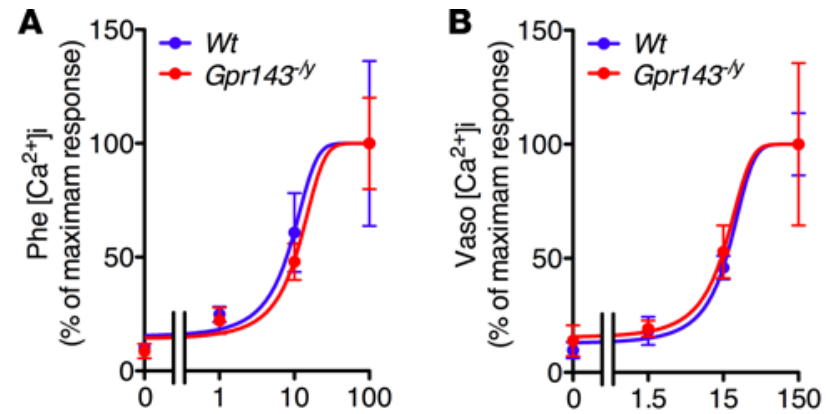

Concentration [Phe $\mu \mathrm{M}$ ]
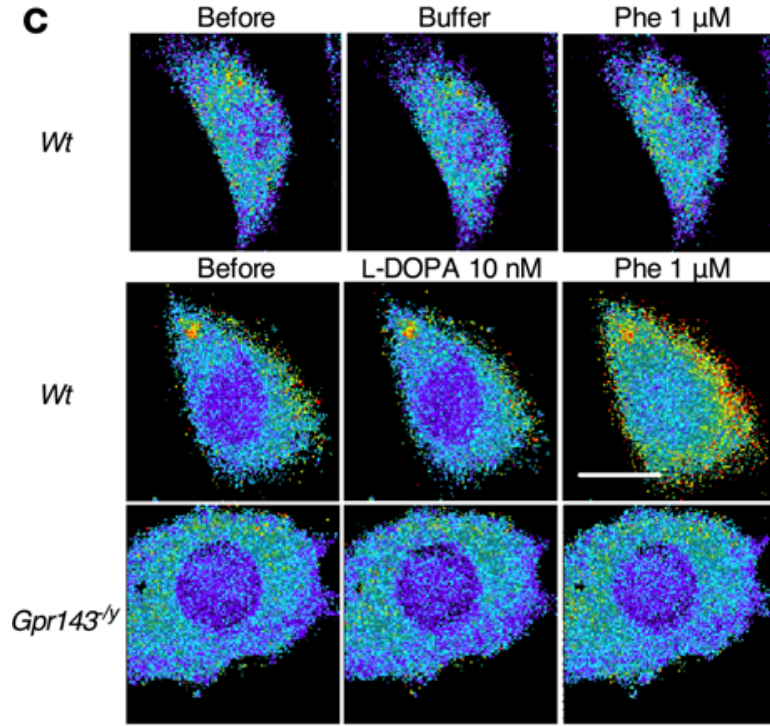

Phe $1 \mu \mathrm{M}$
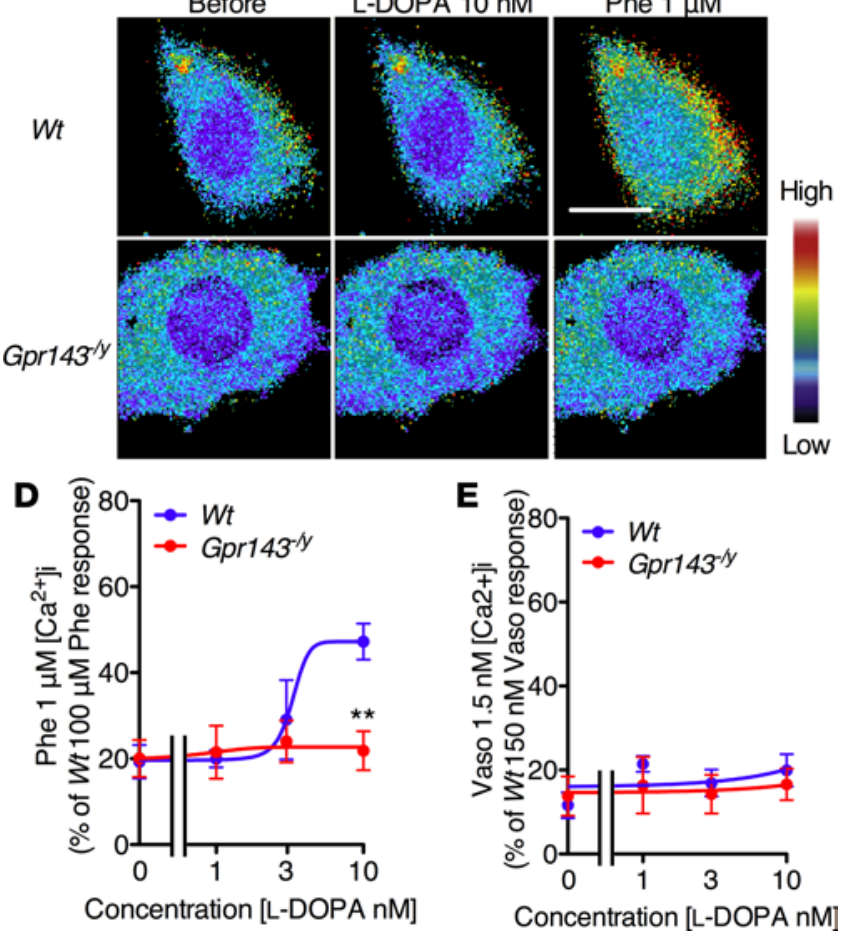

Concentration [Vaso $\mathrm{nM}]$

Figure 3. L-DOPA augments the intracellular $\mathrm{Ca}^{2+}$ response to phenylephrine through GPR143. Intracellular $\mathrm{Ca}^{2+}$ response in VSMCs. (A) Concentration-response curve of phenylephrine-induced (Phe-induced) intracellular $\mathrm{Ca}^{2+}$ increase in WT and Gpr143-1y VSMCs ( $n=9-17$ cells/3-6 dishes). (B) Concentration-response curve of vasopressin-induced (Vaso-induced) intracellular $\mathrm{Ca}^{2+}\left(\left[\mathrm{Ca}^{2+}\right] \mathrm{i}\right)$ increase in WT and Gpr143-/y VSMCs ( $n=6-9$ cells $/ 3-4$ dishes). L-DOPA-induced augmentation of $\mathrm{Ca}^{2+}$ response in VSMCs. (C) Representative images of cytosolic $\mathrm{Ca}^{2+}$ increase detected with Fura-2 in WT and Gpr143-1y VSMCs before and after phenylephrine in the presence of L-DOPA or control buffer. Scale bar: $25 \mu \mathrm{m}$. The magnitude of Fura-2 fluoresce $(340 / 380 \mathrm{~nm})$ intensity is expressed by pseudocolor in a linear scale. (D) Intracellular $\mathrm{Ca}^{2+}$ increase to phenylephrine $(1 \mu \mathrm{M})$ in the presence of L-DOPA in WT and Cpr143-1/ VSMCs ( $n=7-12$ cells/4-6 dishes). (E) Intracellular $\mathrm{Ca}^{2+}$ increase to vasopres$\sin (1.5 \mathrm{nM})$ in the presence of L-DOPA in WT and Gpr143-/y VSMCs ( $n=6-16$ cells/3-6 dishes). All values are mean $\pm S E M$. ${ }^{* *} P<0.01$, compared with $10 \mathrm{nM}$ L-DOPA in WT, 2-way ANOVA with Bonferroni's multiple comparisons test.

with or without phenylephrine (Figure 4, A-C). Indeed, MLC2 and ERK phosphorylation is causally related to ADRA1-mediated contraction (19-22). Because phenylephrine-induced pressor response was

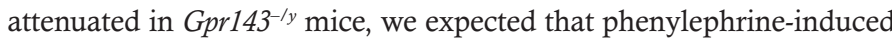

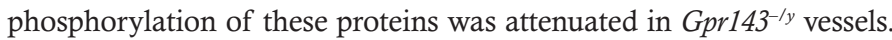
Phenylephrine increased the levels of pMLC2 and pERK in the WT

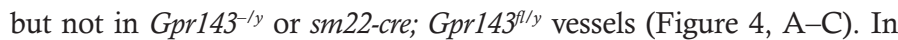
cultured VSMCs, phenylephrine also increased the levels of pERK in WT VSMCs, but not in Gpr143-/y VSMCs (Figure 4D), although

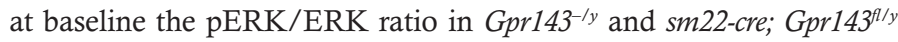
VSMCs was higher than that in WT VSMCs. The mechanisms of

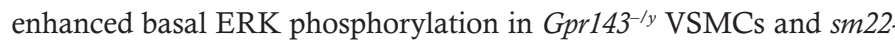
cre; Gpr143//y vessel was unknown. It might be possible that lacking GPR143 in VSMCs activates some compensatory mechanisms directed toward normalizing vasomotor tone of baseline. Even though, the functional interaction between GPR143 and ADRA1s was observed in VSMCs and in intact vessels (Figure 4).

To further confirm the involvement of GPR143 in ADRA1B-mediated signaling, we further investigated the phenylephrine-induced ERK phosphorylation in rat VSMCs (23). As in the case with mouse VSMCs, punctate GPR143 signals were observed in the $\alpha$-smooth muscle actin-positive cells of cultured rat VSMCs (Supplemental Figure 6, A-D). We examined the effect of shRNA knockdown of GPR143 on phenylephrine-induced ERK phosphorylation. The immunofluorescence of GPR143 was decreased in the shgpr143adenovirus-infected VSMCs compared with scramble RNA-adenovirus-infected VSMCs (Supplemental Figure 6, E-I). The expression levels of ADRA1B in membrane were not modified in the shgpr143-adenovirus-infected VSMCs compared with scramble RNA-adenovirus-infected VSMCs (Supplemental Figure 6, J-P). Phenylephrine increased the levels of pERK in the scramble RNA-infected VSMCs, but not in the shgpr143-adenovirus-infected VSMCs (Supplemental Figure 6Q).

GPR143 selectively promotes ADRA1B-mediated ERK phosphorylation. Because each subtype of ADRA1 (ADRA1A, ADRA1B, and ADRA1D) expressed in the VSMCs plays crucial roles in mediating sympathetic neurotransmission (23-25), the attenuated pressor response to phenylephrine in Gpr143-y mice suggests a functional interaction between GPR143 and these ADRA1 subtypes. Thus, GPR143 may interact and functionally couple with ADRA1, thereby modulating the phenylephrine-induced responses. As was expected, GPR143 was physically associated with ADRA1A, ADRA1B, and ADRA1D in immunoprecipitation assays (Figure 5A and Supplemental Figure 7, A and B).

It was reported that ADRA1 stimulation induces ERK activation (26-29). To further examine the functional interaction between GPR143 and ADRA1s, we performed a phosphorylation assay of ERK. 
A

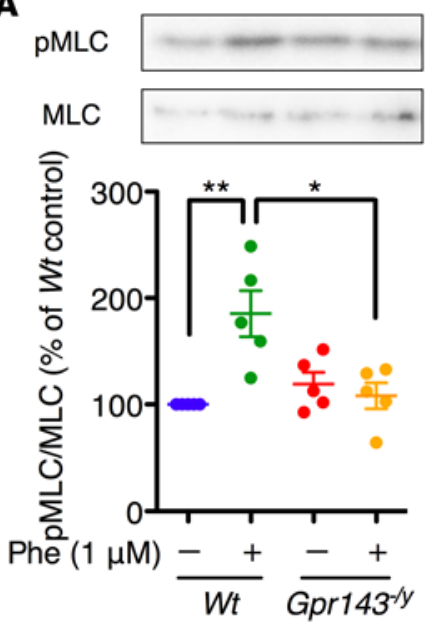

\section{D}

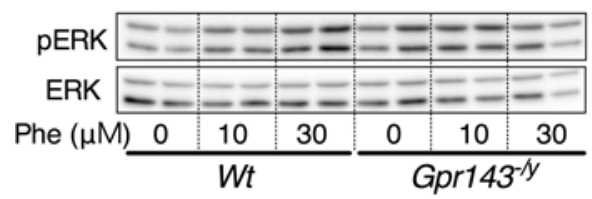

B
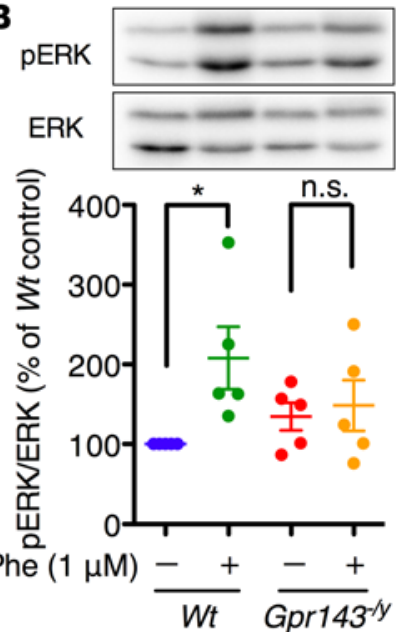

E
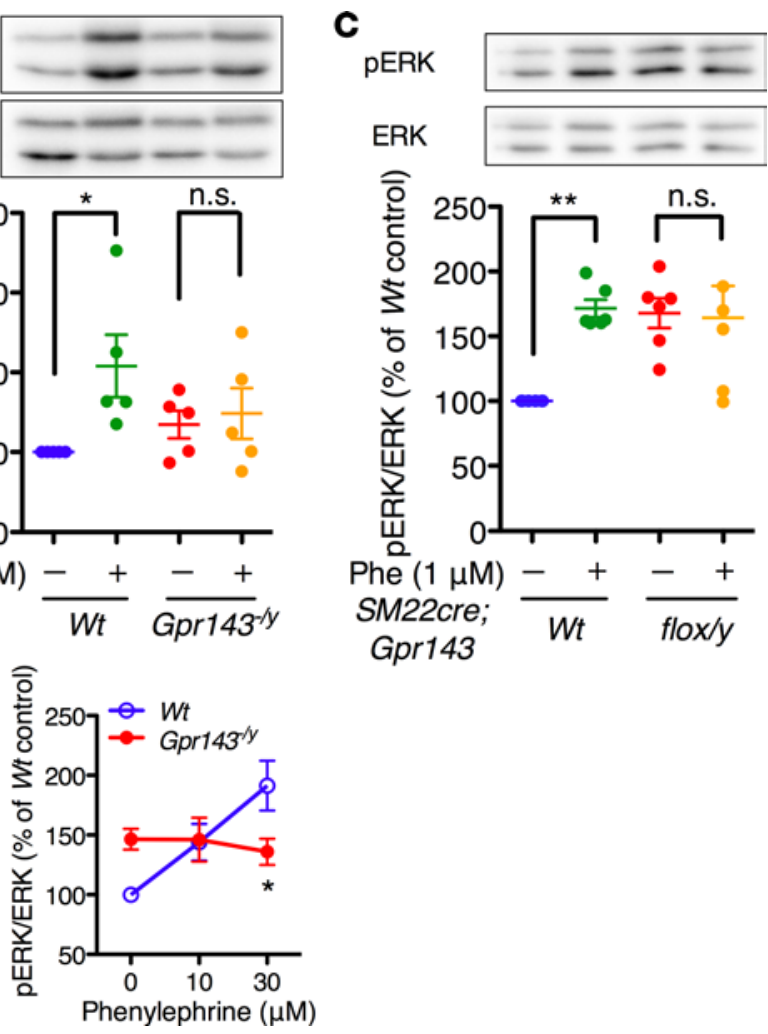

Figure 4. Phenylephrine induces myosin light chain 2 (MLC2) and ERK phosphorylation in the dissected blood vessels and cultured VSMCs of Gpr143-/y mice. Phenylephrine-induced (Phe-induced) myosin light chain 2 (MLC2) and ERK phosphorylation in dissected blood vessels. Protein levels of pMLC2 and MLC2 (A) and pERK and ERK (B and C) were detected after 3-minute treatment with phenylephrine in the dissected vessels from WT and Gpr143 $3^{-1 / y}$, or

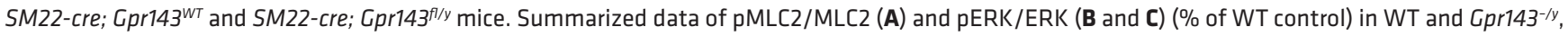
or SM22-cre; Gpr143 ${ }^{W T}$ and SM22-cre; Gpr143 ${ }^{f / y}$ mice $(n=5-6)$ are shown. ${ }^{*} P<0.05,{ }^{*} P<0.01,1$-way ANOVA with Tukey's multiple comparisons test. Phenylephrine-induced ERK phosphorylation in VSMCs. (D) Protein levels of pERK and ERK were determined after 10-minute treatment with phenylephrine in WT and Gpr143-/y mice. (E) Summarized data of pERK/ERK (\% of WT control) $\left(F_{1,42}=7.803, n=8\right) .{ }^{*} P<0.05$, compared with $30 \mu \mathrm{M}$ phenylephrine in WT mice, 2-way ANOVA with Bonferroni's multiple comparisons test. n.s., not significant.

Phenylephrine $(0.1,0.3$, and $1 \mu \mathrm{M})$ stimulation was associated with a modest increase in pERK in Myctagged ADRA1A, ADRA1B, and ADRA1D (ADRA1A-, ADRA1B-, and ADRA1D-Myc) and free EGFPexpressing HEK293 cells. In contrast, phenylephrine markedly increased the pERK in cells expressing GPR143-EGFP and ADRA1B-Myc in a concentration-dependent manner (Figure 5, B and C). Regardless of its physical interactions, GPR143 did not augment phenylephrine-induced ERK phosphorylation in cells coexpressing ADRA1A- or ADRA1D-Myc.

We next examined ligand-dependent interaction between GPR143 and ADRA1B. The immunoprecipitation assay revealed that L-DOPA $(10 \mathrm{nM})$ enhanced the interaction between ADRA1B and GPR143 in HEK293 cells coexpressing GPR143-HA and ADRA1B-Myc (Figure 5D). We then examined the direct interaction between GPR143 and ADRA1B. For this purpose, we performed microscopic analysis using total internal reflection fluorescence (TIRF) microscopy. TIRF microscopic images revealed that GPR143-EGFP was colocalized with ADRA1B-mCherry in the transmembrane of live HEK293 cells (Figure 5E). We also performed Förster resonance energy transfer (FRET) analysis in live HEK293 cells expressing CFP-fused protein (FRET donor) and Venus-fused protein (FRET acceptor). We transfected HEK293 cells with genes encoding Venus-tagged ADRA1B (ADRA1B-Venus) and CFP-tagged GPR143 (GPR143-CFP), free-Venus and GPR143-CFP, or ADRA1B-Venus and free-CFP. The fluorescence spectrum, when the fluorescence protein was excited at a wavelength of $405 \mathrm{~nm}$, revealed emission around $540 \mathrm{~nm}$ in cells expressing both GPR143-CFP and ADRA1B-Venus, but not GPR143-CFP and free-Venus (Supplemental Figure 8, A and B). The fluorescence intensity of GPR143-CFP increased after photobleaching of ADRA1B-Venus, but not free-Venus (Supplemental Figure 8, C and D). FRET signals at significant levels were detected in live HEK293 cells expressing Adra1b-Venus and GPR143-CFP, compared with 
A

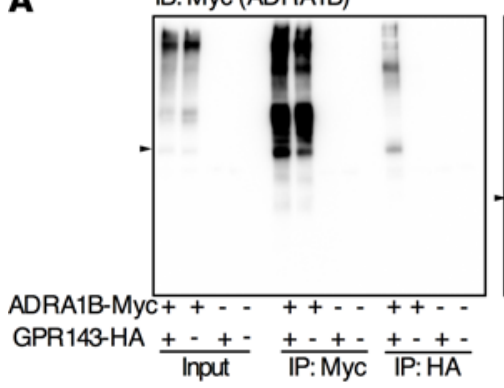

D

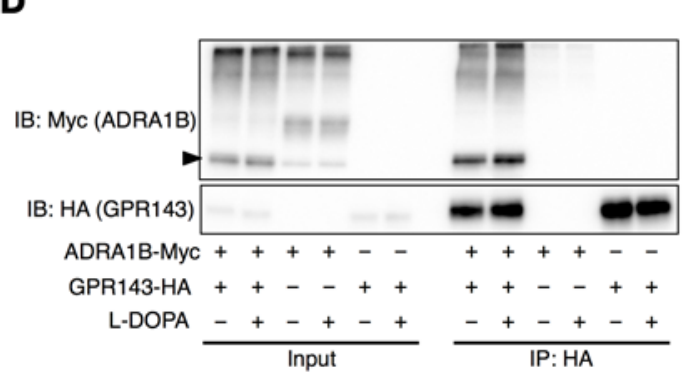

$\mathbf{F}$

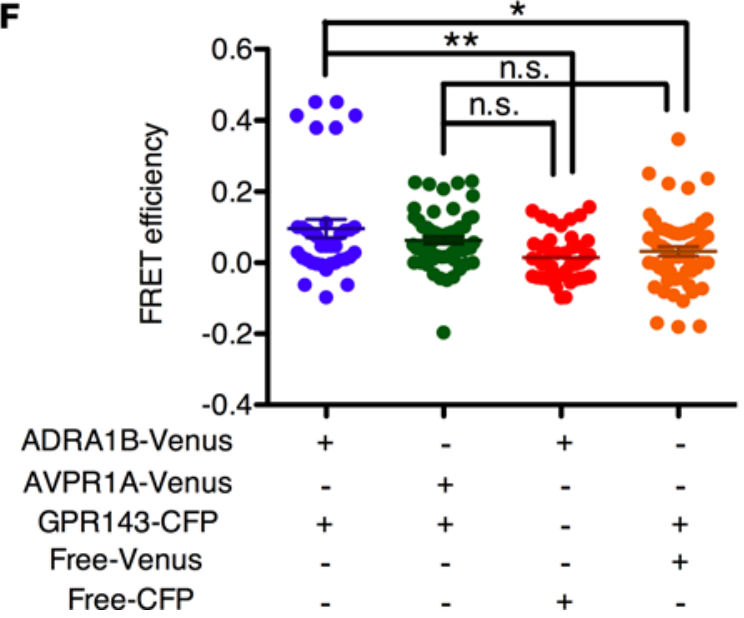

B

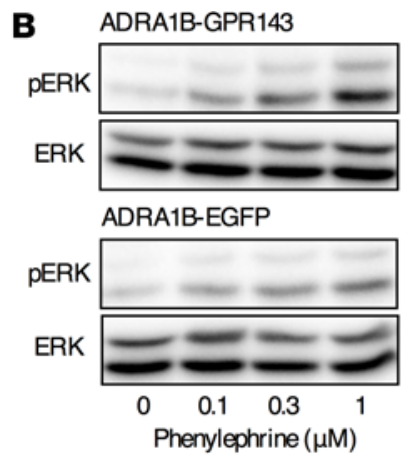

E
ADRA1B-mCherry
IB: HA (GPR143)

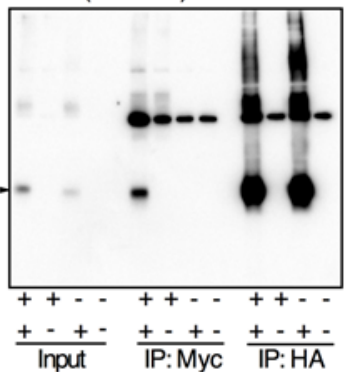

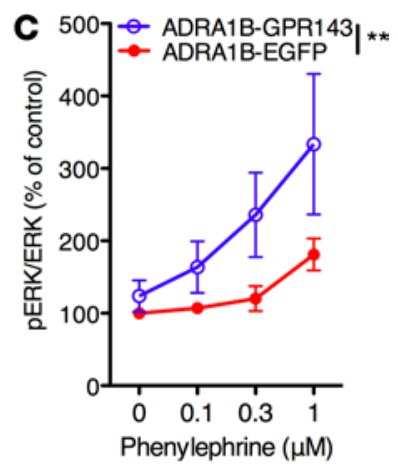
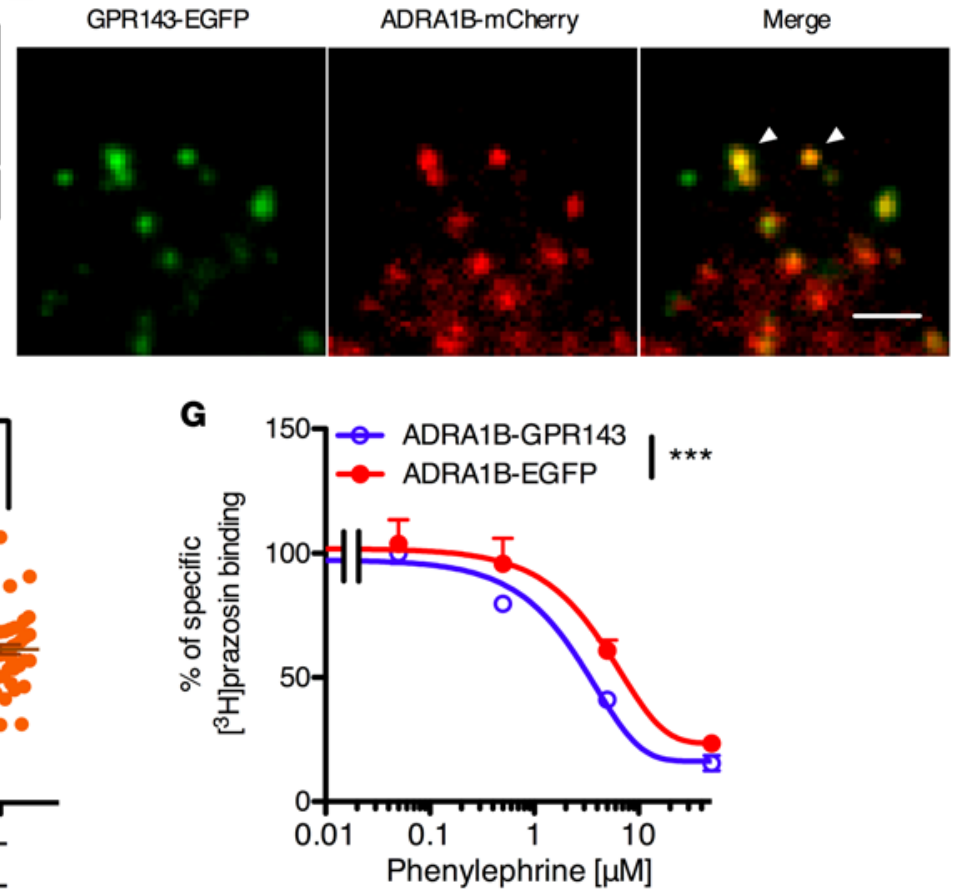

Figure 5. Interaction between GPR143 and ADRA1B. (A) Co-immunoprecipitation (Co-IP) of ADRA1B-Myc and GPR143-HA. Bands were detected by anti-Myc (left) or by anti-HA (right) antibodies. (B) Protein levels of pERK and whole ERK 10 minutes after the treatment with phenylephrine. (C) Summarized data of pERK/ERK (\% of control) in HEK293 cells coexpressing ADRA1B-Myc and free-EGFP or ADRA1B-Myc and GPR143-EGFP $\left(F_{1,32}=7.899, P<0.001, n=5\right)$ (D) Interaction between ADRA1B-Myc and GPR143-HA in the presence or absence of L-DOPA. Note that the interaction was enhanced in the presence of L-DOPA for 1 minute. The arrowhead indicates predicted ADRA1B. (E) TIRF microscopic images of GPR143-EGFP (left), ADRA1B-mCherry (center), and merged images (right) as indicated by white arrowheads ( $n=3$, independent experiments). Scale bar: $3.5 \mu \mathrm{m}$. (F) FRET efficiency between Venus and CFP at the plasma membrane. ${ }^{*} P<0.05$, ${ }^{* *} P<0.01$, 1-way ANOVA with Bonferroni's multiple comparisons test $(n=36-59)$. n.s., not significant. (C) Displacement binding curve of $\left[\mathrm{H}^{3}\right]$-prazosin $(0.4 \mathrm{nM})$ by phenylephrine in HEK293 cells expressing ADRA1B-Myc and GPR143-EGFP or ADRA1B-Myc and free-EGFP $\left(F_{1,40}=19.31\right.$, $\left.{ }^{* *} P<0.001, n=3\right)$. Two-way ANOVA with Bonferroni's multiple comparisons test.

free-Venus and GPR143-CFP or ADRA1B-Venus and free-CFP (Figure 5F). We also confirmed that FRET signals were present in cells expressing ADRA1B-CFP and GPR143-Venus compared with AVPR1A-CFP and GPR143-Venus or free-CFP and GPR143-Venus (Supplemental Figure 9). These results clearly indicate that GPR143 selectively formed a heteromeric complex with ADRA1B in live cells. Because oligomerization of GPCRs may also modulate their ligand-binding properties (30), we investigated whether coexpression of GPR143 affected binding properties of phenylephrine against specific binding of $\left[{ }^{3} \mathrm{H}\right]$-prazosin in ADRA1B-expressing HEK293 cells. The binding affinity of phenylephrine against the specific binding of $\left[{ }^{3} \mathrm{H}\right]$-prazosin was higher in HEK293 cells coexpressing both ADRA1B-Myc and GPR143-EGFP $\left(K_{i}=\right.$ $2.4 \mu \mathrm{M}, n=3)$ than in cells coexpressing ADRA1B-Myc and free-EGFP $\left(K_{i}=9.3 \mu \mathrm{M}, n=3\right)$ (Figure $\left.5 \mathrm{G}\right)$. To investigate whether endogenous GPR143 interacted with Adra1b, we performed immunostaining using 
GPR143
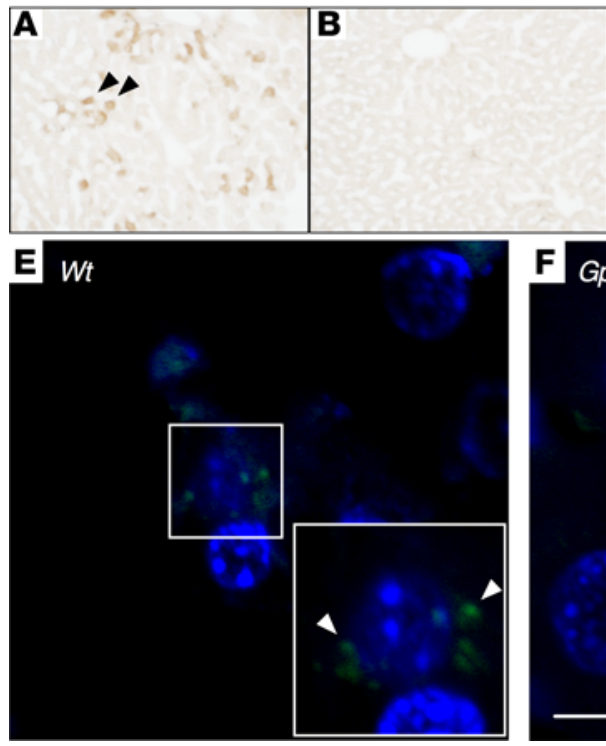

ADRA1B

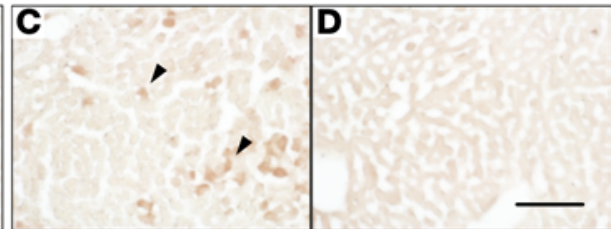

F Gr143.y

Figure 6. Immunohistochemical staining of endogenous GPR143 and ADRA1B. Expression of GPR143 (A) and ADRA1B (C) in the liver (arrowheads). No GPR143 signals were observed in the liver of Gpr143 $3^{-1 y}$ mice (B). No ADRA1B signals were observed in sections incubated with the antigenic peptide-preadsorbed antibody (D). Scale bar: $100 \mu \mathrm{m}$. Endogenous interaction between GPR143 and ADRA1B. (E) The GPR143-ADRA1B interacting signals (green) were detected by in situ proximity ligation assay technology in WT liver (arrowheads). (F) No interacting signals were observed in Gpr143 $3^{-1 / y}$ liver ( $n=2$, independent experiments). The nucleus was counterstained by DAPI (blue). Scale bars: $5 \mu \mathrm{m}$.

in situ proximity ligation assay (PLA). We confirmed the specific immunoreactive signals of both GPR143 and ADRA1B in the liver (Figure 6, A-D). GPR143-ADRA1B interacting signals were detected in WT

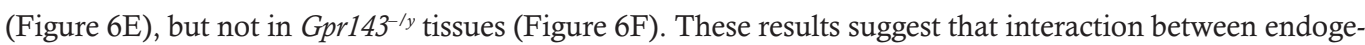
nous GPR143 and ADRA1B potentiates the signaling through ADRA1.

Phasic blood pressure control was lacking in Gpr143-1/ mice. We questioned whether the GPR143 system was involved in phasic and daily blood pressure control. Because the sympathetic nervous system is easily modified by nociceptive stimuli and continuously activated during the active phase, we examined the tail-pinch-induced pressor response and day- and nighttime blood pressure $(31,32)$ in WT and Gpr143-/y mice (Figure 7). The tail-pinch procedure elevated blood pressure in anesthetized WT mice, and the rise was suppressed by pretreatment with prazosin (Figure 7, A and B), indicating that the nociceptive stimulation induced a pressor response through activating the sympathetic nervous system. A tail-pinch-induced

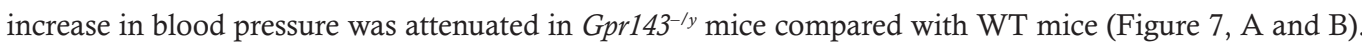
The tail-pinch-induced increase in blood pressure was also attenuated in sm22-cre; Gpr $143^{\text {I/y }}$ mice compared with sm22-cre; Gpr143 ${ }^{W T}$ mice (Figure 7C). We further monitored blood pressure in conscious mice using a radiotelemetry procedure before, during, and after tail-pinch. The mean blood pressure before and after tail-pinch was $108.1 \pm 9.5$ and $155.1 \pm 19.5 \mathrm{mmHg}$ in sm22-cre; Gpr143 ${ }^{W T}$, and $105.8 \pm 6.6$ and $121.6 \pm$ $4.1 \mathrm{mmHg}$ in sm22-cre; Gpr143//y mice, respectively (Figure 7D). The tail-pinch-induced increase in blood

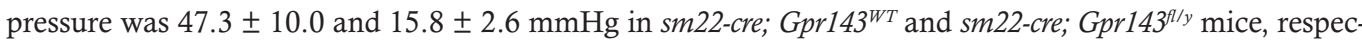
tively. This result indicates that the changes in blood pressure before and after tail-pinch were attenuated in sm22-cre; Gpr143//y mice. It is unlikely that this impaired pressor response is due to nociceptive dysfunction, because there was no difference between WT and Gpr143-1y mice assessed by hot plate test (Supplemental Figure 10). This finding further suggests that GPR143 in the VSMCs is involved in the pressor response to nociceptive stimulation.

To evaluate the role of GPR143 in daily blood pressure control, we measured blood pressure difference

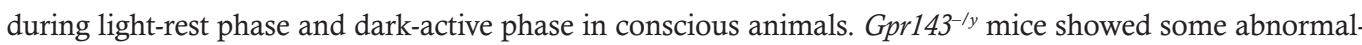

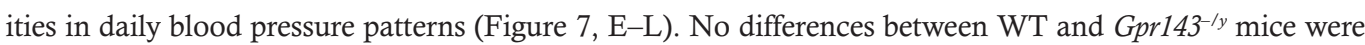
observed in blood pressure levels during the light phase. An increase in blood pressure during the dark-active phase was attenuated in Gpr143-/y mice when compared with WT mice using the tail cuff procedure (Figure 7F). 
A
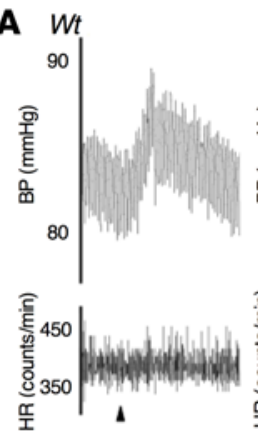

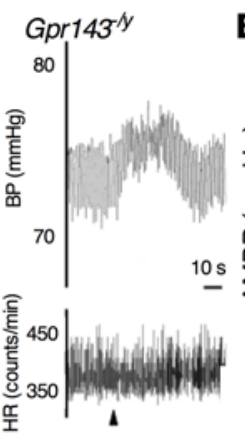

B

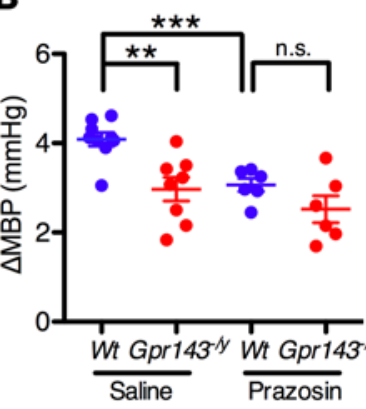

C

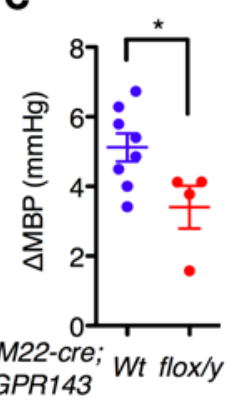

D

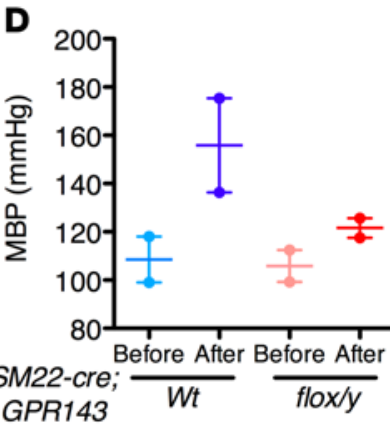

E
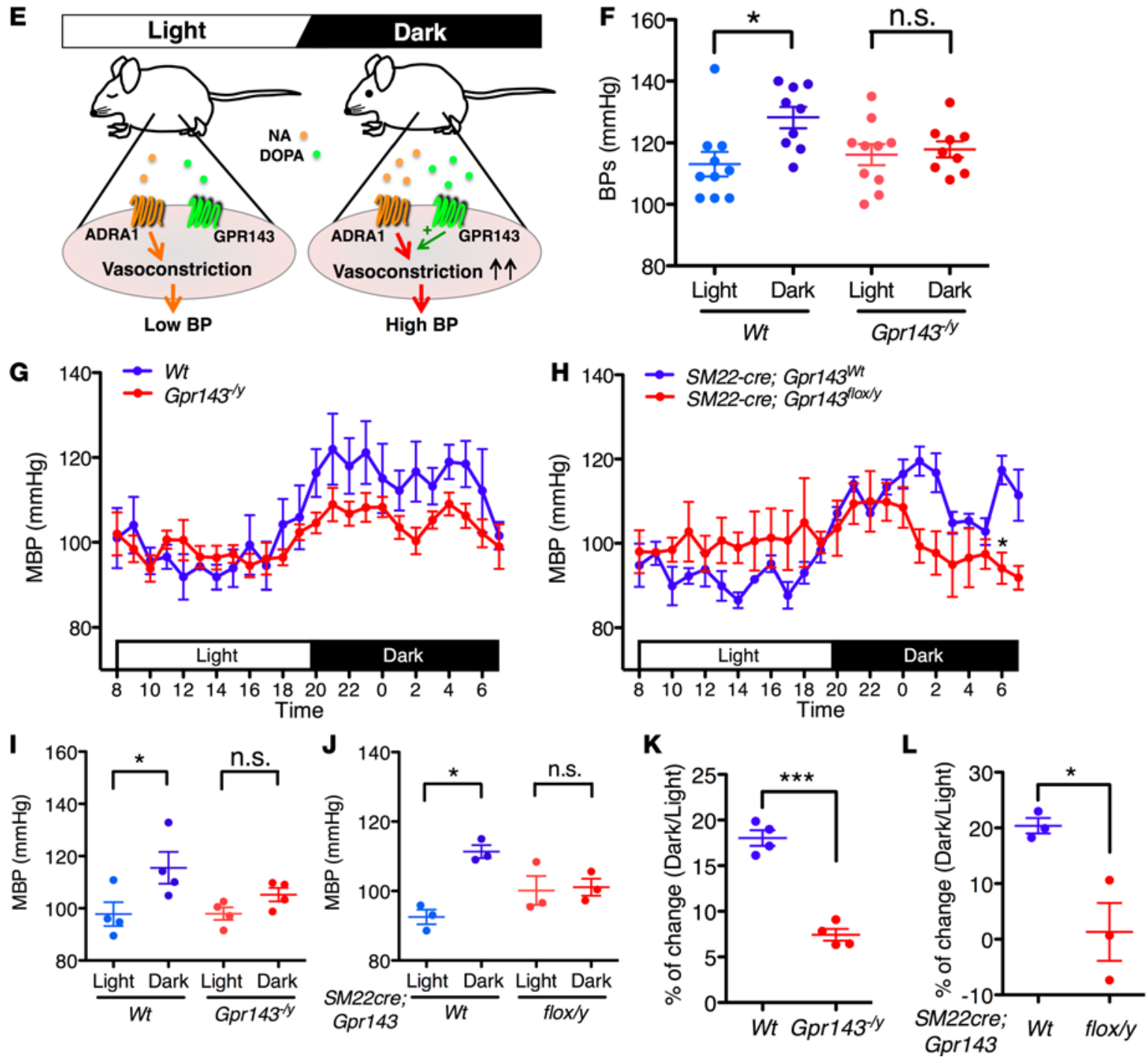

Figure 7. GPR143 is required for arterial BP control under physiological conditions. Tail-pinch-induced pressor response in WT, Gpr143-1y, and SM22cre; Gpr143 ff/y anesthetized and conscious mice. (A) Typical trace of the effects of tail-pinch (10 seconds) on BP and heart rate (HR) in anesthetized WT (left) and Gpr143-1y (right) mice using invasive BP and HR monitoring. (B) Summarized effects of prazosin (1 mg/kg, i.p.) on tail-pinch-induced pressor response in anesthetized WT and Gpr143-/y mice $\left(n=9\right.$ and 6). (C) Tail-pinch-induced pressor response in anesthetized SM22-cre; Gpr143 ${ }^{W T}$

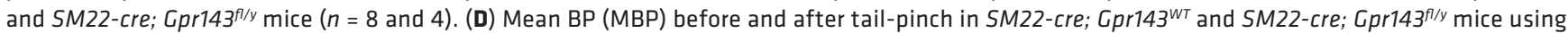
the radiotelemetry method $(n=2)$. Circadian BP during the light and dark phases in conscious WT, Gpr143-1y, SM22-cre; Gpr143 ${ }^{W T}$, and SM22-cre; Gpr143 $3^{f / y}$ mice. (E) An L-DOPA/GPR143 signaling mechanism that regulates blood pressure by sensitizing vascular ADRA1. NA, noradrenaline. (F) Systolic BP (BPs) in WT and Gpr143-1y mice during the light and dark phase using the tail-cuff method $(n=9-10)$. Circadian pattern of MBP ( $\mathbf{G}$ and $\mathbf{H})$; summarized average of MBP during the light and dark phases (I and $\mathbf{J})$; and the percent of change in MBP from the light to dark phases (K and $\mathbf{L})$ in mice using the radiotelemetry method $(n=3-4) .{ }^{*} P<0.05,{ }^{* *} P<0.01,{ }^{* * *} P<0.001$, 1-way ANOVA with Tukey's multiple comparisons test (B, F, I, and J), 2-way ANOVA with Bonferroni's multiple comparisons test ( $\mathbf{G}$ and $\mathbf{H}$ ), or 2-tailed unpaired Student's $t$ test (C, $\mathbf{K}$, and $\mathbf{L})$. n.s., not significant. 
We also performed blood pressure measurement using the radiotelemetry procedure in $G p r 143^{-1 y}$, sm22-cre; Gpr $143^{f / y}$, and corresponding control mice (Figure 7, G and H). The changes in blood pressure from the light-rest to dark-active phase was attenuated in both Gpr143- ${ }^{-1 y}$ and sm22-cre; Gpr $143^{f / y}$ when compared with control mice (Figure 7, G-L). This observation was correlated with the change in blood pressure during the dark-active phase monitored by the tail cuff method (Figure 7F). Because L-DOPA is an endogenous ligand for GPR143 and is released from sympathetic neurons and adrenal medullae in an activity-dependent manner $(3-5,33)$, it is possible that the disruption in day and night blood pressure variation in $G p r 143^{-/ y}$ mice might be due to dysfunction of the sympathetic nervous system. To examine possible involvement of sympathetic activity, we measured plasma levels of L-DOPA, noradrenaline (NA), and adrenaline (Ad) in these mice during light and dark phases. Higher plasma levels of L-DOPA, NA, and Ad were seen in the

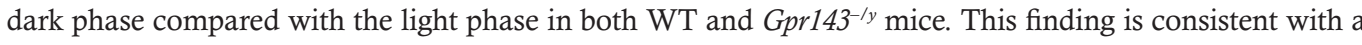
previous report that genes relevant to catecholamine synthesis are under the control of the molecular clock, the deletion of which results in loss of circadian mean arterial rhythms in mice (34). Also, no difference occurred in the plasma levels of these substances between WT and Gpr143-/y mice (Supplemental Figure 2C). In addition, no difference was found in the mRNA levels of Gpr143 and Adra1b during the dark phase relative to during the light phase in WT mice. The fold changes in the mRNA levels of Gpr143 and Adra1b in WT mice during the dark phase relative to the light phase were $1.05 \pm 0.17$ and $1.32 \pm 0.15$, respectively ( $n=6, P>0.05$, unpaired Student's $t$ test). Thus, our findings suggest that endogenous L-DOPA plays a role in regulating daily blood pressure through GPR143 (Figure 7E).

\section{Discussion}

Vasomotor tone is regulated by the sympathetic nervous system through the release of NA, which stimulates ADRs in the blood vessels (35). Here, we demonstrated that L-DOPA itself modulates sympathetic transmission for control over cardiovascular functions. The pressor response to phenylephrine was decreased by $54 \%$ to $61 \%$ in $G$ pr 143 gene-deficient $G p r 143^{-1 y}$ mice compared with WT mice in vivo. Comparable attenuation, $52 \%$ to $68 \%$, was observed in sm22-cre; Gpr $143^{f / y}$ mice. Contractile response to phenylephrine was consistently attenuated in blood vessels isolated from $G$ pr143- $3^{-y}$ mice compared with those from WT mice. When coexpressed with ADRA1B, but not with ADRA1A or ADRA1D, GPR143 augmented the phenylephrine-induced ERK phosphorylation. L-DOPA at noneffective concentrations augmented the phenylephrine-induced contractile response and intracellular $\mathrm{Ca}^{2+}$ response in WT mice. In addition, the phasic blood pressure response to nociceptive stimuli and blood pressure control during the light and dark

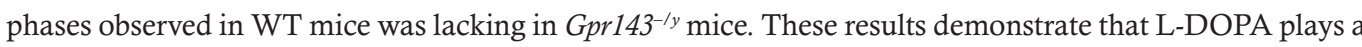
physiological role in regulating blood pressure through coupling between GPR143 and ADRA1 in VSMCs.

GPR143 may possibly modify the function of ADRA1B through crosstalk between downstream signaling cascades of these receptors without direct receptor-receptor interaction. However, several lines of evidence support the formation of a GPR143-ADRA1B complex. First, FRET signal in live HEK293 cells expressing ADRA1B-Venus and GPR143-CFP, but not those expressing AVPR1A-CFP and GPR143-Venus, was detected (Figure 5F). Second, the immunoprecipitation assay revealed L-DOPA-dependent interaction between GPR143 and ADRA1B (Figure 5D). Third, the displacement assay showed high-affinity binding sites for phenylephrine with the coexpression of ADRA1B and GPR143 (Figure 5G). Furthermore, an interaction between endogenous GPR143 and ADRA1B was detected. These results supported our idea that direct protein interaction between GPR143 and ADRA1B potentiates the signaling through ADRA1B. Indeed, the detection of oligomerization of endogenous GPCRs and its physiological relevance have been challenged in recent years. For example, growth hormone secretagogue receptor 1A (GHSR1A) was demonstrated to form heteromers with the dopamine receptor D2 (DRD2) in hypothalamic neurons through labeling of endogenous GHSR $_{1 \mathrm{a}}$ and DRD2 with red-ghrelin and an antibody against the DRD2, respectively (36). In addition, the use of GPCR heteromer disruption may provide a clue to the receptor-receptor interaction. A peptide from the DRD1 physically disrupts DRD1-DRD2 receptor heteromers and inhibits their downstream $\mathrm{Ca}^{2+}$-mediated signaling (37). Further studies are needed to examine whether or not oligomer formation between GPR143 and ADRA1B is required for the modification of the ADRA1B-mediated signaling.

We demonstrated that GPR143 is required for efficient phosphorylation of ERK downstream of ADRA1B activation (Figures 4 and 5). GPR143 augmented phenylephrine-induced ERK phosphorylation in HEK293 cells expressing GPR143 and ADRA1B, but not those with ADRA1A or ADRA1D, compared with those expressing ADRA1B alone (Supplemental Figure 7). This selective augmentation of ADRA1B signaling by 
GPR143 is strongly consistent with previous reports on preferential activation of the ERK cascade by ADRA1B $(27,38)$, although our findings do not exclude the possibility that GPR143 functionally coupled with ADRA1A or ADRA1D as well. Such changes in the receptor's functional property are reminiscent of allosteric modulation of intrinsic efficacy of a ligand of one of the protomers by the other protomer in the GPCR heteromer (39). In the glutamate receptor, metabotropic 2-serotonin receptor 2A (GRM2-HTR2A) receptor heteromer, glutamate induces a stronger GRM2-mediated signaling and serotonin produces a weaker serotonin HTR2Amediated signaling compared with when each receptor is expressed alone. In the same receptor heteromer, the HTR2A antagonist clozapine also induces a positive allosteric modulation of the intrinsic efficacy of glutamate (40). Examples also exist for changes of functional selectivity in G protein coupling in receptor heteromers, such as the DRD1 histamine receptor $\mathrm{H} 3$ heteromer, for which DRD1 receptor agonists activate Gi instead of Gs proteins (39). As another example, heteromerization with GHSR1A modifies DRD2 signaling, resulting in $\mathrm{G}_{\beta \gamma}$-dependent mobilization of $\mathrm{Ca}^{2+}(36)$.

L-DOPA and its receptor GPR143 may contribute to the physiological regulation of blood pressure under nociceptive stress and during light and dark phases (Figure 7). The first step in catecholamine biosynthesis is the formation of L-DOPA from tyrosine by $\mathrm{TH}$, the rate-limiting enzyme of catecholamines. The activity of TH is enhanced by excitation of catecholaminergic neurons (41-43). Thus, L-DOPA may exert a priming effect through GPR143 by sensitizing cardiovascular ADRA1B for the proper control of cardiovascular function (Figure 7E). Our study implies that a disturbance of the L-DOPA/GPR143 mechanism may lead to abnormalities in the circulatory system, which are related to morning hypertension, non-dipper, riser, arrhythmia, and other cardiovascular disorders. Further understanding of the physiological function of L-DOPA will contribute to a new therapeutic approach for the treatment of cardiovascular dysfunctions.

\section{Methods}

Supplemental Methods are available online with this article.

Reagents. Urethane, dopamine, phenylephrine, and prazosin were purchased from Sigma-Aldrich. L-DOPA was purchased from Nacalai Tesque. Vasopressin was purchased from Tocris Bioscience. L-DOPA CHE was synthesized at Drug Discovery Laboratories, Research Institute, Kyowa Hakko Kogyo $(5,8)$. BODIPY-prazosin was purchased from Thermo Scientific. $\left[\mathrm{H}^{3}\right]$-prazosin was purchased from PerkinElmer. Isoflurane was purchased from DS Pharma Animal Health.

Experimental animals. Throughout the experimental procedures, all efforts were made to minimize the num-

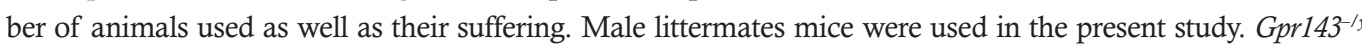
and $G$ pr $143^{f l}$ mice were established as described previously (12). Mice lacking GPR143 in VSMCs were generated by mating female Gpr143 $3^{+/ f}$ and male SM22-cre mice. SM22-cre mice were purchased from the Jackson Laboratory as previously described (16). All mice in the C57BL/6J genetic background weighing 20-25 g were used and housed in temperature-controlled $\left(23 \pm 1^{\circ} \mathrm{C}\right)$ and humidity-controlled $(55 \%)$ rooms. They were maintained on a 12-hour light/12-hour dark cycle with laboratory mouse food and water available ad libitum.

Invasive blood pressure and heart rate monitoring. Mice were anesthetized with urethane $(1.2 \mathrm{~g} / \mathrm{kg}$, i.p.) The femoral artery and vein were cannulated for recording blood pressure and heart rate and for i.v. infusion as previously described (8). Cardiovascular responses to i.v. infusion of phenylephrine $(0.3-2.4 \mu \mathrm{g} /$ $\mathrm{kg})$, vasopressin $(0.25-2.0 \mu \mathrm{g} / \mathrm{kg})$, and L-DOPA CHE $(1-20 \mathrm{mg} / \mathrm{kg})$ were monitored by measuring blood pressure and heart rate. Pretreatment with L-DOPA CHE $(10 \mathrm{mg} / \mathrm{kg}$, i.v. $)$ was performed 5 minutes prior to phenylephrine. To monitor cardiovascular responses to tail-pinch stress, the mice were anesthetized with $3 \%$ isoflurane and thereafter with $2 \%$ isoflurane for maintenance of anesthesia. After cannulation through femoral artery, we performed tail-pinch by pressing the mouse tail root with artery clamp for 10 seconds. Blood pressure and heart rate were recorded before and after the tail-pinch.

Tail-cuff blood pressure monitoring. Systolic blood pressure was measured by sphygmomanometer (BP monitor MK-2000; Muromachi Kikai Co.) using the tail-cuff method as previously described (16). A mouse was introduced into a holder, and blood pressure was monitored under non-anesthetized condition. Blood pressure estimated during 8:30-11:30 a.m. was regarded as the light-phase blood pressure and that estimated during 8:30-11:30 p.m. was regarded as the dark-phase blood pressure.

Hot plate test. The hot plate test was conducted by placing the mouse on a metal surface maintained at $54 \pm 0.1^{\circ} \mathrm{C}$ as described previously (44). The hot plate was surrounded by a transparent plastic barrier. The latency to jumping off the plate or licking a hind paw was recorded. One minute was used as the cutoff time to protect the paw against injury. 
Blood pressure measurement by radiotelemetry. Direct blood pressure measurement was performed by a radiotelemetric method as previously described (16). A blood pressure transducer (PA-C10, Data Sciences International) was inserted into the left carotid artery. Seven days after surgery, mice were housed in a standard cage individually on a receiver under a 12-hour light/12-hour dark cycle. The tail-pinch test was performed by pressing the mouse tail root with artery clamp for 10 seconds. Direct blood pressure was recorded every 5 minutes by radiotelemetry.

Measurement of plasma L-DOPA and catecholamines. Mice were anesthetized with isoflurane, and plasma was collected transcardially during the light phase at 11:00 am and during the dark phase at 9:00 pm. Isoproterenol of $10 \mathrm{ng}$, an internal standard, and trichloroacetic acid-EDTA (TCA-EDTA; final concentration: TCA, 4\%, EDTA, $0.1 \%$ ), were added to plasma, which was incubated for 30 minutes on ice to remove proteins. After centrifugation, L-DOPA and catecholamines in the supernatants were partially purified with activated alumina and eluted with $0.1 \mathrm{~N} \mathrm{HCl}$, and were measured by high performance liquid chromatography with electrochemical detection (Eicom).

Isometric tension measurement of vascular rings of the descending aortas and mesenteric arteries. After WT or Gpr143- $3^{-1 y}$ mice were anesthetized with isoflurane, vessel rings of the descending aortas and mesenteric arteries were prepared and placed in a tissue bath to maintain $37^{\circ} \mathrm{C}$. Two tungsten wires $(40 \mu \mathrm{m}$ in diameter) were threaded into the lumen, and the vessels were mounted in a two-channel myograph (Dual Wire Myograph System-410A, DMT). After the force reached a new steady state, phenylephrine (10 nM to 10 $\mu \mathrm{M})$ was applied to the vascular rings. When used, L-DOPA CHE ( $1 \mathrm{mM})$ was applied 5 minutes prior to phenylephrine. L-DOPA (1 and $10 \mathrm{nM}$ ) was applied 2.5 minutes before phenylephrine under the inhibition of AADC with 3-hydroxybenzylhydrazine $(10 \mu \mathrm{M})$. At the end of all experiments, phenylephrine was washed out, and vasoconstriction was induced by potassium-enriched solutions ( $\mathrm{NaCl}, 22 \mathrm{mM} ; \mathrm{KCl}, 120$ $\mathrm{mM} ; \mathrm{CaCl}_{2}, 1.5 \mathrm{mM}$; glucose, $6 \mathrm{mM} ; \mathrm{MgCl}_{2}, 1 \mathrm{mM}$; HEPES, $5 \mathrm{mM}$; $\mathrm{pH}$ 7.4) to confirm the viability.

Real-time reverse-transcriptase PCR. Total RNAs of mouse descending aortas, heart, and lower brain stem were extracted using TRIzol reagents (Invitrogen) according to the standard protocol for RNA extraction. To obtain first-strand cDNA, $500 \mathrm{ng}$ of the total RNAs were mixed with 50 pmol oligo(dt $)_{20}$, $10 \mathrm{pmol}$ dNTP mixture in a $20-\mu \mathrm{l}$ volume, incubated at $65^{\circ} \mathrm{C}$ for 5 minutes, and chilled on ice for 1 minute. Then, the mixture was supplemented with First-Strand Buffer, $5 \mathrm{mM}$ dithiothreitol, and $100 \mathrm{U}$ SuperScript Reverse Transcriptase (Invitrogen); it was then incubated at $50^{\circ} \mathrm{C}$ for 50 minutes and subsequently at $70^{\circ} \mathrm{C}$ for 15 minutes. The cDNA template produced from each tissue was PCR-amplified using One-Step SYBR PrimeScript RT-PCR KIT II (Takara Bio Inc.), and the assays were run on an ABI 7900HT Fast Real-Time PCR System for semiquantitative analysis of Adra1a, Adra1b, Adra1d, and Gpr143 mRNA. GAPDH was used as an internal control. The cDNA was amplified using the following primers: sense 5'-tcttccatgccccagggat-3' and antisense 5'-ctagacttcctccccgttttcacc-3' for Adra1a, sense 5'-agtagcccagccagaacacta-3' and antisense 5'-gaaggaaatgtccaactccaa-3' for Adra1b, sense 5'-ttgggccgctacagagacc- $3^{\prime}$ and antisense $5^{\prime}$-tttggatccgaaggcagaatc- $3^{\prime}$ for Adra1d, sense $5^{\prime}$-acccaatcctgtttcacaagaca-3' and antisense 5'-ggcagtcattgtttcccactgaat-3' for Gpr143, sense 5'-tggcaaagtggagattgttgcc-3' and antisense $5^{\prime}$-aagatggtgatgggcttcccg- $3^{\prime}$ for GAPDH. Real-time PCR was conducted at $95^{\circ} \mathrm{C}$ for 20 seconds, followed by 45 cycles of $95^{\circ} \mathrm{C}$ for 5 seconds and at $60^{\circ} \mathrm{C}$ for 30 seconds. Results were calculated by the relative $2^{-\Delta \Delta \mathrm{Ct}}$ method using RQ Manager 1.2 software (Invitrogen). All analytical data for Gpr $143^{-/ y}$ mice are presented as fold change compared with WT control mice.

Plasmid construction. The entire coding region of mouse GPR143 was constructed previously (9). Mouse Adra1a, Adra1b, and Adra1d and polycistronic Adra1b-Myc and Gpr143-mCherry were amplified with the primers listed below. The Adra1a, Adra1b, and Adra1d genes were inserted into pc3.1 mycHisA vector (Thermo Scientific), and expression plasmid for Adra1b-Myc and Gpr143-mCherry was likewise inserted into psNha vector. psNha vector was provided by $\mathrm{H}$. Usui (45). All cDNAs were verified by sequencing. Adra1s and the expression plasmid were amplified using the following primers: sense $5^{\prime}$-atcgaattcaccATGGTGCTTCTTTCTGAAAATGCTTCT-3' and antisense 5'-actctcgagGACTTCCTCCCCGTTTTCACCGAG-3' for Adra1a, sense 5'-atcgaattcaaaATGAATCCCGATCTGGACACCGGC-3' and antisense 5'-atctctagaAAAGTGCCCGGGCGCCAGGGGCAT-3' for Adra1b, sense 5'-atcgaattcgagATGACTTTCCGCGACATCCTG-3' and antisense 5'-atctctagaAATGTCAGTCTCCCGGAGGTTGCTGAG-3' for Adra1d, sense 5'-actgaattccgaATGGCCTCCCCGCGCCTGGGAATTTTC-3' and antisense 5'-actatcgattttaaaCTTGTACAGCTCGTCCATGCCGCC-3' for Gpr143-mCherry, sense 5'-actatcgatGGAAGCGGAGCTACTAACTTCAGCCTGCTGAAGCAGGCTGGAGACGTGGAGGAGAAC- 
CCTGGACCTATGAATCCCGATCTGGACACCGGCC-3' and antisense 5'-ACTGGATCCcagatcetcttctgagatgagttttgttcgctacctccgccaccAAAGTGCCCGGGCGCCAGGGGCATGT-3' for Adralb-Myc.

Primary culture of VSMCs from mouse descending aortas. Mouse VSMCs were cultured as previous

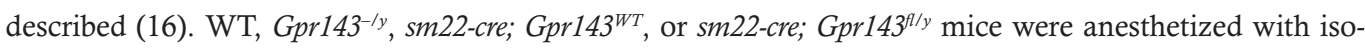
flurane. The abdominal aortas were incised at the middle for blood drainage, and then perfused with $1 \mathrm{ml}$ of saline containing $1,000 \mathrm{U} / \mathrm{ml}$ of heparin from the left ventricle. The descending aortas were dissected out from the rest of aortas. The vessel was cut lengthwise and placed in a 60-mm culture dish. The aortas were cut into pieces that were approximately 2-3 mm each. The aortas were allowed to dry briefly, and then DMEM with 10\% FBS was added gently; the cells were placed in an incubator and left undisturbed for approximately 10 days. The cells were used at passages $2-4$.

Primary culture of rat descending aorta smooth muscle cells. VSMCs in primary culture were obtained from the descending aorta of Wistar rat embryos at E21 as described previously (46). To test the effects of shRNA against GPR143 (8), the cells were transfected with gpr143-adenovirus or scramble-adenovirus vector. After 72-96 hours, the cells were prepared for immunocytochemical and immunoblot analysis.

Co-immunoprecipitation. HEK293 cells (RIKEN BRC) were cotransfected with ADRA1A-Myc, ADRA1B-Myc, or ADRA1D-Myc and GPR143-HA using FuGENE6 (Promega). After 2-day incubation, cells were lysed in $300 \mu \mathrm{l}$ immunoprecipitation buffer $(20 \mathrm{mM}$ Tris- $\mathrm{HCl}, \mathrm{pH}$ 8.0, $150 \mathrm{mM} \mathrm{NaCl}, 1 \mathrm{mM}$ EDTA, $10 \mathrm{mM} \mathrm{NaF}, 1 \mathrm{mM} \mathrm{Na}_{3} \mathrm{VO}_{4}, 0.1 \%$ Nonidet P-40, and $0.1 \%$ protease inhibitor). The lysates were sonicated and centrifuged at 20,400 $\mathrm{g}$ for 20 minutes at $4^{\circ} \mathrm{C}$. The supernatant was incubated with agarose-conjugated anti-Myc (sc-40 AC, Santa Cruz Biotechnology Inc.) and anti-HA antibodies (sc-7392 AC, Santa Cruz Biotechnology Inc.) for 3.5 hours at $4^{\circ} \mathrm{C}$. After being washed 3 times with immunoprecipitation buffer, the samples were detected by immunoblot with anti-Myc (sc-789, Santa Cruz Biotechnology Inc.) and anti-HA (sc-805, Santa Cruz Biotechnology Inc.).

Immunoblot analysis. Aortic vessels were collected with or without $1 \mu \mathrm{M}$ phenylephrine treatment for 3 minutes at $37^{\circ} \mathrm{C}$. Vessels were quickly frozen with liquid nitrogen and soaked with dry ice/acetone containing $10 \%$ TCA, $5 \mathrm{mM} \mathrm{NaF}$, and $10 \mathrm{mM}$ DTT. The samples were thawed to room temperature and washed in acetone containing $10 \mathrm{mM}$ DTT. Proteins were extracted at room temperature in buffer containing $10 \mathrm{mM}$ DTT, $8 \mathrm{M}$ urea, $20 \mathrm{mM}$ Tris- $\mathrm{HCl}$ ( $\mathrm{pH} 8.8$ ), and $22 \mathrm{mM}$ glycine, as previously described (47). Protein concentration was quantified by the Bradford protein assay (Bio-Rad). Briefly, equal amounts of protein $(30 \mu \mathrm{g})$ dissolved in SDS $4 \times$ sample buffer containing DTT $(50 \mu \mathrm{M})$ were separated by SDS-PAGE $(12 \%)$ and then transferred to polyvinylidene fluoride membranes (Millipore). For cultured cells, HEK293 cells or VSMCs were collected after phenylephrine treatment for 10 minutes at $37^{\circ} \mathrm{C}$ and were lysed in immunoprecipitation buffer $(20 \mathrm{mM}$ Tris-HCl, pH 8.0, $150 \mathrm{mM} \mathrm{NaCl}, 1 \mathrm{mM}$ EDTA, $10 \mathrm{mM} \mathrm{NaF}, 1 \mathrm{mM} \mathrm{Na}_{3} \mathrm{VO}_{4}, 0.1 \%$ Nonidet P-40, and 0.1\% protease inhibitor). The lysates were centrifuged at $20,400 \mathrm{~g}$ for 20 minutes at $4^{\circ} \mathrm{C}$. Protein concentration was quantified by the BCA protein assay (Thermo Scientific). Briefly, equal amounts of protein (15 $\mu \mathrm{g})$ dissolved in SDS $4 \times$ sample buffer containing DTT $(50 \mu \mathrm{M})$ were separated by SDS-PAGE $(9 \%)$.

The membranes were cut between 25 and $37 \mathrm{kDa}$ to detect ERK $(42,44 \mathrm{kDa})$ and MLC2 $(18 \mathrm{kDa})$ The samples were then used for immunoblot analysis of anti-ERK (\#9102, diluted 1:6,000), pERK (\#9101, diluted 1:6,000), MLC2 (\#8505, diluted 1:1,000), or pMLC2 (\#3674, diluted 1:1,000) (Cell Signaling Technology) antibodies. After being probed with the primary antibodies, the membrane was washed and incubated with the secondary anti-rabbit IgG antibody coupled to HRP (GE Healthcare). The antibody-antigen complexes were identified with Luminata Forte Western chemiluminescent HRP substrate (Millipore).

Immunochemistry. For immunohistochemistry, the descending aorta and liver sections from mice were postfixed with 4\% paraformaldehyde/PBS pH 7.4 (4 \% PFA) at room temperature for 10 minutes. Sections were then soaked in $0.1 \%$ Triton X-100/PBS (PBST) for 10 minutes and $0.3 \% \mathrm{H}_{2} \mathrm{O}_{2}$ for 30 minutes at room temperature. Following this process, sections were blocked for 1 hour in Triton-TBS (137 mM NaCl, $2.68 \mathrm{mM}$ $\mathrm{KCl}, 25 \mathrm{mM}$ Tris base, $0.1 \%$ Triton X-100 [TBST]) containing 10\% normal goat serum (NGS). The sections were incubated with anti-TH (\#22941, ImmunoStar) antibody, anti-GPR143 antibody (12), or anti-ADRA1B (\#AAR-018, Alomone Labs) antibody at $4^{\circ} \mathrm{C}$ for 24 hours. Antibody was incubated with antigen at $37^{\circ} \mathrm{C}$ for 30 minutes for pre-absorption test. The descending aorta sections were incubated with Dako EnVision+ Dual Link System peroxidase at room temperature for 30 minutes. The liver sections were incubated with anti-rat or anti-rabbit antibody coupled with biotin (Thermo Scientific) at room temperature for 2 hours, and then with $\mathrm{ABC}$ peroxidase kits (Thermo Scientific) at room temperature for 2 hours. Specific labeling was visualized with a ImmPACT DAB Peroxidase Substrate kit (Vector Laboratories). The GPR143-ADRA1B 
interactions were visualized by Duolink In Situ PLA kits (Olink Bioscience) according to the manufacturer's protocol. Specific signals were analyzed using Olympus FV1000 confocal laser scanning microscopy.

For immunocytochemistry, the cultured mouse VSMCs were fixed with 4\% PFA at room temperature for 10 minutes. The cells on the glass-bottom dish were soaked with PBST for 10 minutes and then with $0.3 \% \mathrm{H}_{2} \mathrm{O}_{2}$ for 30 minutes at room temperature. The cells were blocked for 1 hour in TBST containing $10 \%$ NGS. The cells were incubated with anti-GPR143 (sc135161, Santa Cruz Biotechnology Inc.) antibody at $4^{\circ} \mathrm{C}$ for 24 hours. Specific labeling and visualization were performed as described above. After this procedure, hematoxylin and eosin were used for nuclear staining.

Live-cell multicolor TIRF. TIRF imaging was performed as previously described (48). HEK293 cells were plated on a glass-bottom dish and were transfected with ADRA1B-mCherry and GPR143-EGFP. A total internal reflection fluorescence microscope (Leica AM TIRF MC) equipped with an EM-CCD camera system (ImagEM; Hamamatsu Photonics,); a 100×, 1.46 numerical aperture oil-immersion objective (Leica Microsystems); and 488- and 561-nm lasers. The penetration depth of the evanescent field was $110 \mathrm{~nm}$. Experiments were performed in $0.1 \%$ BSA and $10 \mathrm{mM} \mathrm{HEPES} \mathrm{containing} \mathrm{HBSS} \mathrm{buffer} \mathrm{at} \mathrm{room} \mathrm{temperature.}$

FRET analysis. FRET analysis was performed as previously described (48, 49). HEK293 cell were plated on a glass-bottom dish and were transfected with ADRA1B-Venus, AVPR1A-Venus, or free-Venus and GPR143-CFP or free-CFP. Experiments were performed in $0.1 \%$ BSA and $10 \mathrm{mM}$ HEPES containing HBSS buffer at room temperature. FRET signals were measured using a Leica SP5/TCS confocal laser scanning microscope (Leica Microsystems) equipped with a $100 \times / 1.4$ numerical aperture oil-immersion objective and a low-noise hybrid detector (HyD, Leica Microsystems). CFP was excited with a 458-nm argon laser, and the emission was collected around $485 \mathrm{~nm}$. Venus was excited with a 514-nm argon laser, and the emission was collected around $540 \mathrm{~nm}$. For acceptor bleaching, 50 scan cycles with the 514-nm laser line at maximum intensity were used. Using the Leica Application Suite, FRET efficiency (FRET $\left.{ }_{\text {eff }}\right)$ was calculated from the difference in intensity of the donor CFP before and after acceptor photobleaching. FRET $_{\text {eff }}$ was calculated $(50,51)$ as follows: $F R E T_{e f f}=\left(D_{p o s t}-D_{p r e}\right) / D_{\text {post }}$; where $D_{\text {post }}$ and $D_{p r e}$ are the fluorescence intensities of the donor CFP before and after photobleaching, respectively. For fluorescence spectrum imaging, fluorescent protein was excited with a 405-nm blue diode laser, and the emission was collected through a 450- to 650-nm spectrophotometer prism and sliders.

Receptor binding assay. HEK293 cells coexpressing ADRA1B-Myc and free-EGFP or ADRA1B-Myc and GPR143-EGFP were plated in 10-cm culture dishes. Cells were chilled on ice and then rinsed in cold binding buffer, $25 \mathrm{mM}$ Tris, $150 \mathrm{mM} \mathrm{NaCl}, 5 \mathrm{mM}$ EDTA ( $\mathrm{pH}$ 7.4). Cells were suspended to 0.4 $\times 10^{6} \mathrm{cells} / \mathrm{ml}$ and incubated for 1 hour in the binding buffer supplement with $\left[{ }^{3} \mathrm{H}\right]$-prazosin $(0.4 \mathrm{nM}$, PerkinElmer) and phenylephrine $(0.005-50 \mu \mathrm{M}$, final concentration). The cells were washed with the chilled binding buffer 5 times. The radioisotope signals were measured with a liquid scintillation counter. The specific binding values of $\left[{ }^{3} \mathrm{H}\right]$-prazosin were estimated by subtracting the value of $\left[{ }^{3} \mathrm{H}\right]$-prazosin binding in the presence of $1 \mathrm{mM}$ phenylephrine.

Calcium imaging. Mouse VSMCs were loaded with $2.5 \mu \mathrm{M}$ Fura-2 AM (Wako) for 1 hour at $37^{\circ} \mathrm{C}$ in HBSS with 10 mM HEPES buffer (HBSS-HEPES buffer, pH 7.4). After loading, cells were incubated for 10 minutes for recovery in HBSS-HEPES buffer containing 3-hydroxybenzylhydrazine $(10 \mu \mathrm{M})$, and the AADC inhibitor treatment was continued throughout calcium imaging experiments. Calcium imaging by Fura-2 AM was performed with an Olympus IX71 microscope equipped with a UAPo/340 20× objective. Using a filter wheel, excitation light from a 200-W xenon bulb was passed alternately through 340-nm and 380-nm filters. A 30-nm bandpass filter, centered at $510 \mathrm{~nm}$, was selected for the emitted fluorescence that was passed to a CCD camera (iXon3, Nikon). The ratio of $340 / 380 \mathrm{~nm}$ was calculated by MetaFluor (Molecular Devices). For each experiment, images were taken every 2 seconds. Pretreatment with L-DOPA $(10 \mathrm{nM})$ was initiated at 30 seconds after the start of imaging and was continued throughout the experiments. Phenylephrine $(1 \mu \mathrm{M})$ was added 90 seconds after the start of experiment. At the end of the experiments, $\mathrm{KCl}(10 \mathrm{mM})$ was applied as a positive control. The results obtained from cells showing more than a 2-fold increase in intracellular $\mathrm{Ca}^{2+}$ in response to the $\mathrm{KCl}$ stimulus were selected for data analysis.

Statistics. Results are presented as mean \pm SEM of independent experiments. Statistical analysis was performed for 2 groups by 2-tailed paired or unpaired Student's $t$ test, or for multiple groups by 1-way or 2-way ANOVA followed by Tukey's multiple comparisons test or Bonferroni's multiple comparisons test. All analyses were performed using Prism version 5.0a (GraphPad Software Inc.). $P$ values less than 0.05 were considered statistically significant. 
Study approval. All procedures were performed according to the institutional guidelines outlined by the Institutional Animal Care and Use Committee of the Yokohama City University Graduate School of Medicine and according to the NIH Guide for the Care and Use of Laboratory Animals (National Academies Press, 2011) (approval no. F-A-14-046).

\section{Author contributions}

YG and DM designed the research. YG supervised the experiments, data analysis, and interpretation. DM, MK, AS, YN, YK, YOO, TH, ACA, UY, and HW performed research. DM, TH, FN, UY, HI, TS, KT, SU, and YI analyzed data. DM and YG wrote the manuscript.

\section{Acknowledgments}

We thank T. Okada, H. Makihara, and Y. Sekine for excellent technical assistance; Y. Ichikawa, R. Fujiwara, and E. Fujita for their advice on the measurement of vasomotor function; R. Ishiwata and C. Yanai for establishing the mouse lines; Emeritus Professor Y. Misu for encouragement and constructive input; and H. Hirase for useful comments. This work was supported by Scientific Research (B) (General) (no. 15H04687 to YG); Grant-in-Aid for Young Scientists (B) (no. 15K21273 to DM; no. 15K21271 to MK); Scientific Research (C) (General) (no. 26460707 to YK), and Foundation of Strategic Research Projects in Private Universities from the Ministry of Education, Culture, Sport, Science and Technology (MEXT) of Japan; the Japanese SRF Grant for Biomedical Research (no. 1565 to YG); Uehara Memorial Foundation (no. 201320161 to YG); and the fund for Creation of Innovation Centers for Advanced Interdisciplinary Research Areas Program in the Project for Developing Innovation Systems from MEXT (no. 42890001 to YG).

Address correspondence to: Yoshio Goshima, Department of Molecular Pharmacology and Neurobiology, Yokohama City University Graduate School of Medicine, 3-9 Fukuura, Kanazawa-ku, Yokohama, 236-0004, Japan. Phone: 81.045.787.2595; E-mail: goshima@yokohama-cu.ac.jp.

1. Ng KY, Colburn RW, Kopin IJ. Effects of L-dopa on efflux of cerebral monoamines from synaptosomes. Nature. 1971;230(5292):331-332.

2. Goshima Y, Kubo T, Misu Y. Transmitter-like release of endogenous 3,4-dihydroxyphenylalanine from rat striatal slices. J Neurochem. 1988;50(6):1725-1730.

3. Chritton SL, et al. Adrenomedullary secretion of DOPA, catecholamines, catechol metabolites, and neuropeptides. J Neurochem. 1997;69(6):2413-2420.

4. Tyce GM, Hunter LW, Ward LE, Rorie DK. Effluxes of 3,4-dihydroxyphenylalanine, 3,4-dihydroxyphenylglycol, and norepinephrine from four blood vessels during basal conditions and during nerve stimulation. J Neurochem. 1995;64(2):833-841.

5. Misu Y, Goshima Y. Is L-dopa an endogenous neurotransmitter? Trends Pharmacol Sci. 1993;14(4):119-123.

6. Yue JL, Okamura H, Goshima Y, Nakamura S, Geffard M, Misu Y. Baroreceptor-aortic nerve-mediated release of endogenous L-3,4-dihydroxyphenylalanine and its tonic depressor function in the nucleus tractus solitarii of rats. Neuroscience. 1994;62(1):145-161.

7. Kubo T, Goshima Y, Hata H, Misu Y. Evidence that endogenous catecholamines are involved in $\alpha 2$-adrenoceptor-mediated modulation of the aortic baroreceptor reflex in the nucleus tractus solitarii of the rat. Brain Res. 1990;526(2):313-317.

8. Hiroshima Y, et al. The protein ocular albinism 1 is the orphan GPCR GPR143 and mediates depressor and bradycardic responses to DOPA in the nucleus tractus solitarii. Br J Pharmacol. 2014;171(2):403-414.

9. Bassi MT, et al. Cloning of the gene for ocular albinism type 1 from the distal short arm of the X chromosome. Nat Genet. 1995;10(1):13-19.

10. Schiaffino MV, Tacchetti C. The ocular albinism type 1 (OA1) protein and the evidence for an intracellular signal transduction system involved in melanosome biogenesis. Pigment Cell Res. 2005;18(4):227-233.

11. Lopez VM, Decatur CL, Stamer WD, Lynch RM, McKay BS. L-DOPA is an endogenous ligand for OA1. PLoS Biol. 2008;6(9):e236.

12. Fukuda N, et al. Expression of ocular albinism 1 (OA1), 3, 4- dihydroxy- L-phenylalanine (DOPA) receptor, in both neuronal and non-neuronal organs. Brain Res. 2015;1602:62-74.

13. Koshimizu TA, et al. V1a vasopressin receptors maintain normal blood pressure by regulating circulating blood volume and baroreflex sensitivity. Proc Natl Acad Sci U S A. 2006;103(20):7807-7812.

14. Palmisano I, et al. The ocular albinism type 1 protein, an intracellular G protein-coupled receptor, regulates melanosome transport in pigment cells. Hum Mol Genet. 2008;17(22):3487-3501.

15. Innamorati G, Piccirillo R, Bagnato P, Palmisano I, Schiaffino MV. The melanosomal/lysosomal protein OA1 has properties of a G protein-coupled receptor. Pigment Cell Res. 2006;19(2):125-135.

16. Kobayashi Y, et al. Mice lacking hypertension candidate gene ATP2B1 in vascular smooth muscle cells show significant blood pressure elevation. Hypertension. 2012;59(4):854-860.

17. Lepore JJ, Cheng L, Min Lu M, Mericko PA, Morrisey EE, Parmacek MS. High-efficiency somatic mutagenesis in smooth muscle cells and cardiac myocytes in SM22 $\alpha$-Cre transgenic mice. Genesis. 2005;41(4):179-184. 
18. Kim JH, et al. High cleavage efficiency of a $2 \mathrm{~A}$ peptide derived from porcine teschovirus-1 in human cell lines, zebrafish and mice. PLoS One. 2011;6(4):e18556.

19. Tarjus A, et al. Role of smooth muscle cell mineralocorticoid receptor in vascular tone. Pflugers Arch. 2015;467(8):1643-1650.

20. Xu Q, Liu Y, Gorospe M, Udelsman R, Holbrook NJ. Acute hypertension activates mitogen-activated protein kinases in arterial wall. J Clin Invest. 1996;97(2):508-514.

21. Zhao Z, Wang J, Huo Z, Wang Z, Mei Q. FTY720 elevates smooth muscle contraction of aorta and blood pressure in rats via ERK activation. Pharmacol Res Perspect. 2017;5(3):e00308.

22. Dessy C, Kim I, Sougnez CL, Laporte R, Morgan KG. A role for MAP kinase in differentiated smooth muscle contraction evoked by alpha-adrenoceptor stimulation. Am J Physiol. 1998;275(4 pt 1):C1081-C1086.

23. Cipolletta E, et al. Calmodulin-dependent kinase II mediates vascular smooth muscle cell proliferation and is potentiated by extracellular signal regulated kinase. Endocrinology. 2010;151(6):2747-2759.

24. Hosoda C, et al. Two $\alpha 1$-adrenergic receptor subtypes regulating the vasopressor response have differential roles in blood pressure regulation. Mol Pharmacol. 2005;67(3):912-922.

25. Methven L, Simpson PC, McGrath JC. $\alpha 1 \mathrm{~A} / \mathrm{B}-\mathrm{knockout}$ mice explain the native $\alpha 1 \mathrm{D}$-adrenoceptor's role in vasoconstriction and show that its location is independent of the other $\alpha 1$-subtypes. Br J Pharmacol. 2009;158(7):1663-1675.

26. Huang Y, et al. An $\alpha 1 \mathrm{~A}$-adrenergic-extracellular signal-regulated kinase survival signaling pathway in cardiac myocytes. Circulation. 2007;115(6):763-772.

27. Jensen BC, Swigart PM, Montgomery MD, Simpson PC. Functional $\alpha-1 B$ adrenergic receptors on human epicardial coronary artery endothelial cells. Naunyn Schmiedebergs Arch Pharmacol. 2010;382(5-6):475-482.

28. Pardanaud L, et al. Sympathetic innervation promotes arterial fate by enhancing endothelial ERK activity. Circ Res. 2016;119(5):607-620.

29. Sumi Y, Woehrle T, Chen Y, Yao Y, Li A, Junger WG. Adrenergic receptor activation involves ATP release and feedback through purinergic receptors. Am J Physiol Cell Physiol. 2010;299(5):C1118-C1126.

30. Azdad K, Gall D, Woods AS, Ledent C, Ferré S, Schiffmann SN. Dopamine D2 and adenosine A2A receptors regulate NMDA-mediated excitation in accumbens neurons through A2A-D2 receptor heteromerization. Neuropsychopharmacology. 2009;34(4):972-986.

31. Jackson KL, Palma-Rigo K, Nguyen-Huu TP, Davern PJ, Head GA. Major contribution of the medial amygdala to hypertension in BPH/2J genetically hypertensive mice. Hypertension. 2014;63(4):811-818.

32. Kayaba Y, et al. Attenuated defense response and low basal blood pressure in orexin knockout mice. Am J Physiol Regul Integr Comp Physiol. 2003;285(3):R581-R593.

33. Hunter LW, Rorie DK, Tyce GM. Dihydroxyphenylalanine and dopamine are released from portal vein together with noradrenaline and dihydroxyphenylglycol during nerve stimulation. J Neurochem. 1992;59(3):972-982.

34. Curtis AM, Cheng Y, Kapoor S, Reilly D, Price TS, Fitzgerald GA. Circadian variation of blood pressure and the vascular response to asynchronous stress. Proc Natl Acad Sci U S A. 2007;104(9):3450-3455.

35. Brodde OE, Michel MC. Adrenergic receptors and their signal transduction mechanisms in hypertension. J Hypertens Suppl. 1992;10(7):S133-S145.

36. Kern A, Albarran-Zeckler R, Walsh HE, Smith RG. Apo-ghrelin receptor forms heteromers with DRD2 in hypothalamic neurons and is essential for anorexigenic effects of DRD2 agonism. Neuron. 2012;73(2):317-332.

37. Hasbi A, et al. A peptide targeting an interaction interface disrupts the dopamine D1-D2 receptor heteromer to block signaling and function in vitro and in vivo: effective selective antagonism. FASEB J. 2014;28(11):4806-4820.

38. Goyal R, Goyal D, Chu N, Van Wickle J, Longo LD. Cerebral artery $\alpha-1$ AR subtypes: high altitude long-term acclimatization responses. PLoS One. 2014;9(11):e112784.

39. Ferré S, et al. G protein-coupled receptor oligomerization revisited: functional and pharmacological perspectives. Pharmacol Rev. 2014;66(2):413-434.

40. Fribourg M, et al. Decoding the signaling of a GPCR heteromeric complex reveals a unifying mechanism of action of antipsychotic drugs. Cell. 2011;147(5):1011-1023.

41. Andrews DN, Weiner N. Evidence for the involvement of a cyclic AMP independent protein kinase in the activation of rat striatal tyrosine hydroxylase. Proc West Pharmacol Soc. 1979;22:163-167.

42. Weiner N, Cloutier G, Bjur R, Pfeffer RI. Modification of norepinephrine synthesis in intact tissue dy drugs and during shortterm adrenergic nerve stimulation. Pharmacol Rev. 1972;24(2):203-221.

43. Yamauchi T, Fujisawa H. Evidence for three distinct forms of calmodulin-dependent protein kinases from rat brain. FEBS Lett. 1980;116(2):141-144.

44. Nakamura $\mathrm{H}$, et al. Comprehensive behavioral study and proteomic analyses of CRMP2-deficient mice. Genes Cells. 2016;21(10):1059-1079.

45. Usui H, Taniguchi M, Yokomizo T, Shimizu T. Plexin-A1 and plexin-B1 specifically interact at their cytoplasmic domains. Biochem Biophys Res Commun. 2003;300(4):927-931.

46. Yokoyama U, et al. Multiple transcripts of $\mathrm{Ca}^{2+}$ channel $\alpha 1$-subunits and a novel spliced variant of the $\alpha 1 \mathrm{C}$-subunit in rat ductus arteriosus. Am J Physiol Heart Circ Physiol. 2006;290(4):H1660-H1670.

47. Bae YK, Qin H, Knobel KM, Hu J, Rosenbaum JL, Barr MM. General and cell-type specific mechanisms target TRPP2/ PKD-2 to cilia. Development. 2006;133(19):3859-3870.

48. Kamikubo Y, et al. Functional cooperation of metabotropic adenosine and glutamate receptors regulates postsynaptic plasticity in the cerebellum. J Neurosci. 2013;33(47):18661-18671.

49. Kamikubo Y, Tabata T, Sakairi H, Hashimoto Y, Sakurai T. Complex formation and functional interaction between adenosine A1 receptor and type-1 metabotropic glutamate receptor. J Pharmacol Sci. 2015;128(3):125-130.

50. Kenworthy AK, Edidin M. Distribution of a glycosylphosphatidylinositol-anchored protein at the apical surface of MDCK cells examined at a resolution of < 100 A using imaging fluorescence resonance energy transfer. J Cell Biol. 1998;142(1):69-84.

51. Lippincott-Schwartz J, Snapp E, Kenworthy A. Studying protein dynamics in living cells. Nat Rev Mol Cell Biol. 2001;2(6):444-456. 